\title{
Orbit-orbit relativistic corrections to the pure vibrational non-Born-Oppenheimer energies of $\mathrm{H}_{2}$
}

\author{
Monika Stanke, ${ }^{1, a)}$ Dariusz Kȩdziera, ${ }^{2}$ Sergiy Bubin, ${ }^{1}$ Marcin Molski, ${ }^{3}$ and \\ Ludwik Adamowicz ${ }^{1,4, b)}$ \\ ${ }_{1}^{1}$ Department of Chemistry, University of Arizona, Tucson, Arizona 85721, USA \\ ${ }^{2}$ Department of Chemistry, Nicholas Copernicus University, ul. Gagarina 7, Pb 87-100 Toruń, Poland \\ ${ }^{3}$ Department of Theoretical Chemistry, Faculty of Chemistry, Adam Mickiewicz University, ul. Grunwaldzka \\ 6, Poznań, PL 60-780, Poland \\ ${ }^{4}$ Department of Physics, University of Arizona, Tucson, Arizona 85721, USA
}

(Received 26 October 2007; accepted 21 December 2007; published online 20 March 2008)

\begin{abstract}
We report the derivation of the orbit-orbit relativistic correction for calculating pure vibrational states of diatomic molecular systems with $\sigma$ electrons within the framework that does not assume the Born-Oppenheimer (BO) approximation. The correction is calculated as the expectation value of the orbit-orbit interaction operator with the non-BO wave function expressed in terms of explicitly correlated Gaussian functions multiplied by even powers of the internuclear distance. With that we can now calculate the complete relativistic correction of the order of $\alpha^{2}$ (where $\alpha=1 / c$ ). The new algorithm is applied to determine the full set of the rotationless vibrational levels and the corresponding transition frequencies of the $\mathrm{H}_{2}$ molecule. The results are compared with the previous calculations, as well as with the frequencies obtained from the experimental spectra. The comparison shows the need to include corrections higher than second order in $\alpha$ to further improve the agreement between the theory and the experiment. (c) 2008 American Institute of Physics.
\end{abstract}

[DOI: $10.1063 / 1.2834926$ ]

\section{INTRODUCTION}

The quantum electrodynamics (QED) creates a general theoretical framework for the analysis of the relativistic effects in atoms and molecules in bound states. However, even for systems with a few electrons accurate calculations of the relativistic effects are too expensive to be carried out on the present-day computers. Furthermore, the QED DiracCoulomb (DC) equation is only fully correct for a single electron in the Coulombic field and approximations are introduced when systems with more than one electron are considered. Also an additional problem appears due to the lack of a lower bound for the negative energy spectrum in the DC equation. Faced with those difficulties effort has been made to develop an effective approach to account for the relativistic effects in light atomic and molecular systems based on the perturbation theory. The zeroth-order level in such an approach is the nonrelativistic Schrödinger equation, and the perturbation Hamiltonian representing the relativistic effects is obtained based on the so called nonrelativistic QED theory (NRQED). ${ }^{1,2}$ We should mention that the perturbation approach to account for the relativistic corrections can also be developed without using NRQED as it was shown by Bethe and Salpeter. ${ }^{3}$

In the NRQED theory the relativistic corrections appear as quantities proportional to powers of the fine structure parameter $\alpha$ (where $\alpha=1 / c$ ). Those corrections are determined using the perturbation theory with the nonrelativistic wave

\footnotetext{
a)Permanent address: Department of Physics, Nicholas Copernicus University, ul. Grudziadzka 5, PL 87-100, Toruń, Poland.

b)Electronic mail: ludwik@u.arizona.edu.
}

function as the zero-order function. In addition to the NRQED corrections one can also calculate corrections due to the structure of the nucleus and its polarizability. With those corrections NRQED is at present the most accurate theoretical framework for calculating bound states of light atoms and molecules. In the literature the corrections proportional to $\alpha^{2}$ are often referred to as the relativistic corrections, while those proportional to higher order of $\alpha$ are called QED corrections.

The NRQED approach has been used in very accurate calculations on small atoms and atomic ions. Our contribution to the field has been the recent calculations concerning four electron atomic systems, $\mathrm{Be}$ and $\mathrm{Li}^{-}{ }^{4-6}$ In those works we have used wave functions expanded in terms of Gaussian functions that explicitly depend on the interparticle distances [the so-called explicitly correlated Gaussians (ECG)] and obtained in calculations where the Born-Oppenheimer (BO) approximation is not assumed in the perturbative evaluation of the $\alpha^{2}$ relativistic effects. With those effects added to the nonrelativistic energies and with the QED corrections calculated up to the order of $\alpha^{4}$ by Pachucki and Komasa ${ }^{7-9}$ also included, we obtained results matching the experimental ionizations potentials and transition frequencies within the experimental accuracy.

The non-BO calculations using Gaussians are simpler than molecular calculations. This results from the fact that in an approach that does not assume the $\mathrm{BO}$ approximation regarding the separability of the electronic and nuclear motion in a molecule one not only needs to very accurately describe the electronic correlation effects but also the correlation effects due to the coupled motions of the electrons and 
the nuclei and the nucleus-nucleus correlation effects. These latter effects require that the basis functions used in the calculations include terms that effectively separate the nuclei from each other in the wave functions. In diatomic calculations this is accomplished by including powers of the internuclear distance as premultipliers of the Gaussian functions (we call the Gaussians with the premultipliers the "diatomic Gaussians"). ${ }^{10-15}$ While similar separation also occur for electrons, their small masses make them overlap to much higher degree than it is the case for the much heavier nuclei.

The work on implementation of ECGs in atomic and molecular calculations have been carried out in our group for a number of years. ${ }^{10-15}$ The key aspect of this implementation has been an effective optimization of the nonlinear parameters involved in the Gaussian exponents that in our approach has been carried out variationally with the use of the analytical gradient of the variational energy functional with respect to the parameters.

In this work we report the development and implementation of the algorithm for calculating the orbit-orbit relativistic correction of the order of $\alpha^{2}$ for a diatomic molecule with $\sigma$ electrons determined as the expectation value of the sum of the electronic and nuclear operators representing the orbit-orbit interactions. This follows our previous works, where the algorithms for the mass-velocity and Darwin relativistic corrections were developed. ${ }^{16,17}$ The expectation value is calculated using the non-BO wave function expanded in terms of ECGs. The use of the non-BO framework for determining the orbit-orbit interaction with the explicitly correlated diatomic Gaussians in the present work is a new feature that has never before been implemented beyond the single-electron molecular case. The previous calculations of this interaction have been done assuming the $\mathrm{BO}$ approximation and the calculated effects only concerned the electronelectron contribution. ${ }^{18,19}$

We should also mention that in our recent work ${ }^{21}$ we calculated orbit-orbit corrections for a diatomic system, the $\mathrm{HeH}^{+}$ion, using explicitly correlated Gaussians with complex exponential parameters. While complex Gaussians allow molecular non-BO calculations, they are much less efficient than the diatomic Gaussians particularly in describing excited states. Thus, the calculations of the orbit-orbit corrections described in the mentioned paper were limited to only the lowest three pure vibrational states of $\mathrm{HeH}^{+}$and they were not as accurate as the results obtained in this work.

For the test application of the new algorithm we chose the $\mathrm{H}_{2}$ molecule, which has been a frequently used molecular model for very accurate molecular calculations. In the present calculations we determine the energies of all fifteen pure vibrational states and the corresponding transition frequencies of this system. We should note that the vibrational quantum number is not strictly speaking a good quantum number for a molecular system because, if the BO approximation is not assumed, the vibrational motion of the nuclei couples to some degree with the motion of electrons. Thus, one should more correctly call the states considered in this work the rotationless states since they all correspond to the zero total angular momentum.

In our previous work on $\mathrm{H}_{2}$ (Ref. 20) we used 5000
ECGs in the calculations for the vibrational states of this system. With that many functions the energies of the lowest two to three states have been practically converged. However, for upper states there had been room for improvement. In general, due to the higher number of radial nodes in the wave functions of the higher excited states, these states require more basis functions to be determined with a comparable accuracy as the lower states. Thus in this work, in order to increase the accuracy of the calculated energy levels, apart from including the orbit-orbit corrections to the energies of the 15 states of $\mathrm{H}_{2}$, we also increased the basis set size used for each state from the previously used 5000 to 5800 .

The most complete experimental spectrum of the pure vibrational transitions of $\mathrm{H}_{2}$ are still those of Dabrowski ${ }^{22}$ obtained more than two decades ago. The accuracy of those results is probably not more than $0.1 \mathrm{~cm}^{-1}$. In this work we have made an attempt to obtain more precise estimation of the lowest $\mathrm{H}_{2}$ transition using an extrapolation procedure and the more recently measured rovibrational transitions of this system and its isotopomers. This newly obtained transition is used in this work for comparison with the calculated results.

We start this work with a brief description of the method we use (a more complete description of the method can be found in our recent reviews ${ }^{10,11}$ ). We also describe the procedure used to calculate the orbit-orbit correction. Next we describe the procedure used to obtain a better estimate of the lowest experimental pure vibrational transition frequency. The results obtained in the calculations and their comparison with the experimental results are presented in the last section.

\section{THE METHOD USED IN THE CALCULATIONS}

The approach used here is based on the variational minimization of the total energy of the system expressed as the expectation value of the internal nonrelativistic Hamiltonian $\hat{H}_{\text {nonrel }}$ obtained from the "laboratory frame" Hamiltonian by separating out the center-of-mass motion. For $\mathrm{H}_{2}$ the internal Hamiltonian has the following form:

$$
\begin{aligned}
\hat{H}_{\text {nonrel }}= & -\frac{1}{2}\left(\sum_{i=1}^{3} \frac{1}{\mu_{i}} \nabla_{\mathbf{r}_{i}}^{2}+\sum_{i=1}^{3} \sum_{j \neq i}^{3} \frac{1}{m_{0}} \nabla_{\mathbf{r}_{i}} \cdot \nabla_{\mathbf{r}_{j}}\right) \\
& +\sum_{i=1}^{3} \frac{q_{0} q_{i}}{r_{i}}+\sum_{i<j}^{3} \frac{q_{i} q_{j}}{r_{i j}} .
\end{aligned}
$$

In Eq. (1) $q_{0}=q_{1}=1$ are the charges of the hydrogen nuclei (protons) and $q_{2}=q_{3}=-1$ are the electron charges, $\mathbf{r}_{i}, i$ $=1,2,3$, are the position vectors of one of the protons and the two electrons with respect to the other proton (the so called "reference particle"), $r_{i}$ are their lengths, $r_{i j}=\left|\mathbf{r}_{j}-\mathbf{r}_{i}\right|$, $m_{0}=m_{1}=1836.15267261 m_{e}$ are the masses of the protons, $m_{2}=m_{3}=m_{e}=1$ are the electron masses, ${ }^{23}$ and $\mu_{i}$ $=m_{0} m_{i} /\left(m_{0}+m_{i}\right)$ is the reduced mass of particle $i\left({ }^{\prime}\right.$ denotes the vector transposition). The internal Hamiltonian (1) describes three pseudoparticles with charges equal to the charges of the original particles moving in the central potential of the central field of the charge of the reference proton.

To calculate the relativistic corrections of the order of $\alpha^{2}$ we start with the respective operators representing the mass- 
velocity (MV), Darwin (D), spin-spin (SS), and orbit-orbit (OO) interactions in the laboratory coordinate frame and we transform them to the internal coordinate system of the nine coordinates, $\mathbf{r}_{i}, i=1,2,3$. More details of this transformation for the MV and D corrections can be found in Ref. 20. The transformed MV, D, SS, and OO Hamiltonians have the following form:

$$
\begin{aligned}
\hat{H}_{\mathrm{MV}}= & -\frac{1}{8}\left[\frac{1}{m_{0}^{3}}\left(\sum_{i=1}^{3} \nabla_{\mathbf{r}_{i}}\right)^{4}+\sum_{i=1}^{3} \frac{1}{m_{i}^{3}} \nabla_{\mathbf{r}_{i}}^{4}\right] \\
\hat{H}_{\mathrm{D}}= & -\frac{\pi}{2} \sum_{i=1}^{3}\left(\frac{\left(1+2 \kappa_{0}\right)}{m_{0}^{2}}+\frac{\left(1+2 \kappa_{i}\right)}{m_{i}^{2}}\right) q_{0} q_{i} \delta^{3}\left(r_{i}\right) \\
& -\frac{\pi}{2} \sum_{i=1}^{3} \sum_{j \neq i}^{3} \frac{\left(1+2 \kappa_{i}\right)}{m_{i}^{2}} q_{i} q_{j} \delta^{3}\left(r_{i j}\right) \\
\hat{H}_{\mathrm{SS}}= & 2 \pi \frac{\left(1+\kappa_{2}\right)\left(1+\kappa_{3}\right) q_{2} q_{3}}{m_{2} m_{3}} \delta^{3}\left(r_{23}\right) \\
& +2 \pi \frac{\left(1+\kappa_{0}\right)\left(1+\kappa_{1}\right) q_{0} q_{1}}{m_{0} m_{1}} \delta^{3}\left(r_{1}\right), \\
\hat{H}_{\mathrm{OO}}= & -\frac{1}{2} \sum_{i=1}^{3} \sum_{j=1}^{3} \frac{q_{0} q_{j}}{m_{0} m_{j}}\left[\frac{1}{r_{j}} \nabla_{\mathbf{r}_{i}} \cdot \nabla_{\mathbf{r}_{j}}+\frac{1}{r_{j}^{3}} \mathbf{r}_{j} \cdot\left(\mathbf{r}_{j} \cdot \nabla_{\mathbf{r}_{i}}\right) \nabla_{\mathbf{r}_{j}}\right] \\
& +\frac{1}{2} \sum_{i=1}^{3} \sum_{j>i}^{3} \frac{q_{i} q_{j}}{m_{i} m_{j}}\left[\frac{1}{r_{i j}} \nabla_{\mathbf{r}_{i}} \cdot \nabla_{\mathbf{r}_{j}}\right. \\
& \left.+\frac{1}{r_{i j}^{3}} \mathbf{r}_{i j} \cdot\left(\mathbf{r}_{i j} \cdot \nabla_{\mathbf{r}_{i}}\right) \nabla_{\mathbf{r}_{j}}\right]
\end{aligned}
$$

where $\kappa_{0}=\kappa_{1}=\kappa_{p}=1.7928473565$ and $\kappa_{2}=\kappa_{3}=\kappa_{e}$ $=0.0011596521811$ are the anomalies of the magnetic moments of a proton and an electron, respectively. We should mention that the spin-orbit interactions are zero for $\mathrm{H}_{2}$ due to the singlet multiplicity for both the electrons and the protons. The total relativistic correction was calculated for each state as the expectation value of the Hamiltonian $\hat{H}^{\text {rel }}$ representing all four relativistic corrections,

$$
\hat{H}^{\mathrm{rel}}=\hat{H}_{\mathrm{MV}}+\hat{H}_{\mathrm{D}}+\hat{H}_{\mathrm{SS}}+\hat{H}_{\mathrm{OO}}
$$

with the non-BO wave function.

The non-BO wave functions of the pure rotationless vibrational states of $\mathrm{H}_{2}$ were expanded in terms of one center, spherically symmetric ECGs that included preexponential multipliers consisting of the internuclear distance $r_{1}$, raised to a non-negative even power $m_{k}$. These are the same functions as those used before in our non-BO calculations of other diatomic systems. ${ }^{12-15}$ The functions have the following form:

$$
\phi_{k}=r_{1}^{m_{k}} \exp \left[-\mathbf{r}^{\prime}\left(A_{k} \otimes I_{3}\right) \mathbf{r}\right],
$$

where $\mathbf{r}=\left\{\mathbf{r}_{1}^{\prime}, \mathbf{r}_{2}^{\prime}, \mathbf{r}_{3}^{\prime}\right\}^{\prime}$. In our previous calculations we have demonstrated that these functions are very effective in describing nonadiabatic, zero angular momentum states of diatomic systems with $\sigma$ electrons. The $r_{1}^{m_{k}}$ factor in function (7) enables to generate radial nodes in the wave function and allows a very effective description of the nucleus-nucleus correlation effects. We refer the reader for more information on the Hamiltonian transformation and the selection of the basis functions for diatomic non-BO calculations to our recent reviews. ${ }^{10,11}$ The derivation of the algorithms of the matrix elements with the $\hat{H}_{\mathrm{MV}}, \hat{H}_{\mathrm{D}}$, and $\hat{H}_{\mathrm{SS}}$ operators were described in Ref. 20. The derivation of the matrix elements with the $\hat{H}_{\mathrm{OO}}$ operator is shown in Appendix A.

In the present non-BO calculations of the vibrational spectrum of $\mathrm{H}_{2}$ we used the standard variational method applied separately to each state. The minimization of the Rayleigh quotient with respect to the linear expansion coefficients $c_{k}$, the Gaussian exponential parameters $\left\{A_{k}\right\}$, and the preexponential powers $\left\{m_{k}\right\}$, and generating the wave functions

$$
E=\min \frac{c^{\prime} H\left(\left\{m_{k}\right\},\left\{A_{k}\right\}\right) c}{c^{\prime} S\left(\left\{m_{k}\right\},\left\{A_{k}\right\}\right) c}
$$

has been the most consuming part of the calculations. In the minimization of Eq. (8) with respect to the Gaussian exponential parameters we used the analytical energy gradient which greatly accelerated the process of the wave function optimization. Also, in order to avoid restricting the elements of the $A_{k}$ matrix to make it positive definite, we used the Cholesky-factored form of $A_{k}, A_{k} \equiv L_{k} L_{k}^{\prime}$, where $L_{k}$ was a lower triangular matrix. With such a representation of $A_{k}$, this matrix is automatically positive definite for any real values of the $L_{k}$ matrix elements. In the calculations the $L_{k}$ matrix elements replaced the elements of $A_{k}$ as the optimization variables. The range of the preexponential powers $m_{k}$, used was from 0 to 250 , and all the powers were partially optimized for each state.

The calculations concerned all 15 pure vibrational states of $\mathrm{H}_{2}$. As mentioned, the number of basis functions used for each state was 5800 (by 800 more than in our previous calculations ${ }^{20}$ ). After the non-BO nonrelivistic wave functions were generated for all states, we use them to calculate the relativistic corrections. The nonrelativistic energies corrected for the relativistic effects were used to calculate the transition frequencies.

\section{ESTIMATION OF PURE VIBRATIONAL TRANSITION $v=0 \rightarrow 1$ OF $\mathrm{H}_{2}$ FROM ROTATION-VIBRATIONAL SPECTRA}

As mentioned, in the present work we compare the calculated pure vibrational transition frequencies of $\mathrm{H}_{2}$ with those obtained experimentally by Dabrowski. ${ }^{22}$ Since the work of Dabrowski appeared more than two decades ago there have been some more measurements done concerning the $\mathrm{H}_{2}$ rovibrational spectrum that allow to establish more precise value for the frequency of the $J=0, v=0 \rightarrow 1$ transition. This was done in this work by fitting the line positions of the available rovibrational transitions to the Dunham's energy formula ${ }^{24}$ 
TABLE I. Dunham's spectral parameters $\left(\right.$ in $\mathrm{cm}^{-1}$ ) for the $\mathrm{H}_{2}$ molecule.

\begin{tabular}{lc}
\hline \hline$Y_{\mathrm{ij}}$ & $v=0 \rightarrow 1$ \\
\hline$Y_{10}$ & $4161.1660(3)$ \\
$Y_{11}$ & $-2.95854(18)$ \\
$Y_{12} \cdot 10^{3}$ & $1.430(21)$ \\
$Y_{13} \cdot 10^{6}$ & $2.48(84)$ \\
$Y_{14} \cdot 10^{8}$ & $-3.9(11)$ \\
$Y_{15} \cdot 10^{10}$ & $2.01(67)$ \\
$Y_{16} \cdot 10^{13}$ & $-4.8(18)$ \\
$Y_{17} \cdot 10^{16}$ & $4.4(17)$ \\
$Y_{01}$ & $233.8(126)$ \\
$Y_{02}$ & $-72.2(53)$ \\
$Y_{03}$ & $10.90(79)$ \\
$Y_{04} \cdot 10^{-1}$ & $-7.55(55)$ \\
$Y_{05} \cdot 10^{-2}$ & $2.58(19)$ \\
$Y_{06} \cdot 10^{-4}$ & $-4.23(31)$ \\
$Y_{07} \cdot 10^{-6}$ & $2.63(19)$ \\
$\hat{\sigma}$ & 1.089 \\
$J_{\min }-J_{\max }$ & $0-18$ \\
$\mathrm{Lines}$ & 83 \\
$\nu_{0 \rightarrow 1}$ & $4161.1660(3)$ \\
\hline \hline
\end{tabular}

$$
E_{v J}=\sum_{i, j} Y_{i j}\left(v+\frac{1}{2}\right)^{i}[J(J+1)]^{j}
$$

In Eq. (9) $v=0,1,2 \ldots$ and $J=0,1,2 \ldots$ denote the vibrational and the rotational quantum numbers, respectively. With that the frequency of the $0 \rightarrow 1$ pure vibrational transition is directly evaluated from the relationship

$$
\nu_{0 \rightarrow 1}=Y_{10} \mathrm{~cm}^{-1}
$$

provided that the higher order vibrational parameters $Y_{20}$, $Y_{30}$, etc., are negligibly small.

As the input data for calculating the parameters of the Dunham's expansion we used 83 spectral transitions (including some duplicated lines measured with different preci- sions) reported in Refs. 25-33. The calculations were performed using a weighted non-linear least-square optimization routine with weights taken as the inverse squares of the uncertainties of the experimental data, $u_{i}$, ranging from 0.06 up to $0.00008 \mathrm{~cm}^{-1} \cdot{ }^{25-33}$ To obtain the best fits of Dunham's parameters $Y_{i j}$ from the spectra, we did the following: (i) the number of fitted parameters was minimized and adjusted to be consistent with the minimum value of the normalized standard deviation $\hat{\sigma}$ and the standard deviation $\sigma$, (ii) the estimated standard error $\sigma_{i}$, of the fitted parameter $i$ and of the correlation coefficient, $c c(i, j)$, between parameters $i$ and $j$ was optimized to obtain the best fit. Dunham's parameters obtained in the calculations and the estimate of the $J=0, v=0 \rightarrow 1$ transition are presented in Table I.

\section{THE RESULTS}

In Table II we present the total nonrelativistic energies for all 15 pure vibrational states obtained in the calculations. In parentheses next to each energy value we show by how much the energy decreased by enlarging the basis set from 5000 used in our previous work on $\mathrm{H}_{2}$ (Ref. 20) to 5800. This increase resulted in lowering of the energy for each state by some amount. The largest decrease occurred for the higher states where the convergence in terms of the number of basis functions is slower due to, as mentioned, the larger number of radial nodes. For the first few states the decrease of the energy is marginal showing that for these states the calculations are essentially converged in terms of the number of basis functions. In the second column in Table II we show the nonrelativistic total energies obtained by Wolniewicz. ${ }^{34,35}$ It is interesting to note that, for the three lowest states, our energies are slightly lower than his, but for the other states they are slightly higher. The difference in the approach may contribute to these differences, as well as still not sufficiently

TABLE II. Total nonrelativistic ( $\left.E^{\text {nrel }}\right)$ non-BO energies, their absolute error estimates $\left(\Delta E^{\text {nrel }}\right)$, and relativistic $\left(E^{\text {rel }}\right)$ non-BO energies for pure vibrational states of $\mathrm{H}_{2}$. All values are in hartrees. The relativistic energies were obtained by adding the complete $\alpha^{2}$ corrections to the nonrelativistic energies. In parenthesis by the nonrelativistic energies we show the energy lowering achieved by enlarging the basis set from 5000 to 5800 functions. $\Delta E^{\text {nrel }}$ is an estimate of the absolute error in the total nonrelativistic energy of the state due to the incompleteness of the basis. Also, the nonrelativistic energies of Wolniewicz (Ref. 35) are presented for comparison.

\begin{tabular}{rcccc}
\hline \hline$v$ & $E^{\text {nrel }}$ & $\Delta E^{\text {nrel }}$ & $E^{\text {nrel }}($ Wolniewicz $)$ & $E^{\text {rel }}$ \\
\hline 0 & $-1.16402503063\left(-1.1 \times 10^{-10}\right)$ & $2 \times 10^{-10}$ & -1.1640250185 & -1.16403573658 \\
1 & $-1.14506537154\left(-4.0 \times 10^{-10}\right)$ & $5 \times 10^{-10}$ & -1.1450653629 & -1.14507597714 \\
2 & $-1.12717793442\left(-9.4 \times 10^{-10}\right)$ & $9 \times 10^{-10}$ & -1.1271779324 & -1.12718846076 \\
3 & $-1.11034047725\left(-2.1 \times 10^{-9}\right)$ & $1 \times 10^{-9}$ & -1.1103404855 & -1.11035095118 \\
4 & $-1.09453917065\left(-2.9 \times 10^{-9}\right)$ & $1 \times 10^{-9}$ & -1.0945391940 & -1.09454961931 \\
5 & $-1.07976944298\left(-5.2 \times 10^{-9}\right)$ & $2 \times 10^{-9}$ & -1.0797694803 & -1.07977989507 \\
6 & $-1.06603723158\left(-6.7 \times 10^{-9}\right)$ & $2 \times 10^{-9}$ & -1.0660372849 & -1.06604771800 \\
7 & $-1.05336075621\left(-1.0 \times 10^{-8}\right)$ & $2 \times 10^{-9}$ & -1.0533608258 & -1.05337131302 \\
8 & $-1.04177302990\left(-1.4 \times 10^{-8}\right)$ & $3 \times 10^{-9}$ & -1.0417731139 & -1.04178369409 \\
9 & $-1.03132537329\left(-1.9 \times 10^{-8}\right)$ & $3 \times 10^{-9}$ & -1.0313254708 & -1.03133619138 \\
10 & $-1.02209238425\left(-2.5 \times 10^{-8}\right)$ & $4 \times 10^{-9}$ & -1.0220924876 & -1.02210340850 \\
11 & $-1.01417904848\left(-3.1 \times 10^{-8}\right)$ & $5 \times 10^{-9}$ & -1.0141791536 & -1.01419034240 \\
12 & $-1.00773110060\left(-3.7 \times 10^{-8}\right)$ & $5 \times 10^{-9}$ & -1.0077311979 & -1.00774274235 \\
13 & $-1.00295038538\left(-4.2 \times 10^{-8}\right)$ & $5 \times 10^{-9}$ & -1.0029504633 & -1.00296247763 \\
14 & $-1.00011593436\left(-3.4 \times 10^{-8}\right)$ & $5 \times 10^{-9}$ & -1.0001159762 & -1.00012860548 \\
\hline \hline
\end{tabular}


TABLE III. Relativistic corrections of the order of $\alpha^{2}$ (in hartees) to the non-BO pure vibrational energies of $\mathrm{H}_{2}$.

\begin{tabular}{ccccc}
\hline \hline$v$ & $\begin{array}{c}\text { Mass velocity } \\
\left(\times 10^{-5}\right)\end{array}$ & $\begin{array}{c}\text { Darwin } \\
\left(\times 10^{-5}\right)\end{array}$ & Orbit-orbit & Spin-spin \\
\hline 0 & -8.657713 & 7.298899 & $-2.537587 \times 10^{-6}$ & $5.457613 \times 10^{-6}$ \\
1 & -8.414374 & 7.088578 & $-2.408717 \times 10^{-6}$ & $5.096376 \times 10^{-6}$ \\
2 & -8.189038 & 6.892388 & $-2.281874 \times 10^{-6}$ & $4.754261 \times 10^{-6}$ \\
3 & -7.980607 & 6.709371 & $-2.156050 \times 10^{-6}$ & $4.425130 \times 10^{-6}$ \\
4 & -7.788031 & 6.538816 & $-2.030115 \times 10^{-6}$ & $4.102013 \times 10^{-6}$ \\
5 & -7.610628 & 6.379645 & $-1.902798 \times 10^{-6}$ & $3.786762 \times 10^{-6}$ \\
6 & -7.448063 & 6.231713 & $-1.772583 \times 10^{-6}$ & $3.473719 \times 10^{-6}$ \\
7 & -7.299335 & 6.093825 & $-1.637664 \times 10^{-6}$ & $3.157829 \times 10^{-6}$ \\
8 & -7.164558 & 5.965663 & $-1.495830 \times 10^{-6}$ & $2.840258 \times 10^{-6}$ \\
9 & -7.044081 & 5.847237 & $-1.344278 \times 10^{-6}$ & $2.512031 \times 10^{-6}$ \\
10 & -6.937318 & 5.737515 & $-1.179434 \times 10^{-6}$ & $2.168227 \times 10^{-6}$ \\
11 & -6.844584 & 5.635966 & $-9.966327 \times 10^{-7}$ & $1.801360 \times 10^{-6}$ \\
12 & -6.767008 & 5.542714 & $-7.897333 \times 10^{-7}$ & $1.400629 \times 10^{-6}$ \\
13 & -6.705164 & 5.456952 & $-5.507375 \times 10^{-7}$ & $9.471695 \times 10^{-7}$ \\
14 & -6.660159 & 5.378000 & $-2.715985 \times 10^{-7}$ & $4.250207 \times 10^{-7}$ \\
\hline \hline
\end{tabular}

complete basis sets used in our calculations particularly for higher states. However, as it will be shown later in this work, the small discrepancies between Wolniewicz's and our results have very small effect on the transition energies.

In the last column in Table II we show the total energies obtained by adding the complete $\alpha^{2}$ relativistic corrections to the nonrelativistic energies. Individual contributions to those corrections are shown in Table III. Among those corrections there are two, the mass-velocity and Darwin corrections that change little with the vibrational excitation and there are two, the orbit-orbit and spin-spin corrections, that decrease tenfold from the $v=0$ state to the $v=14$ state. However, since the former two corrections are in average ten times larger than the latter, their relative contributions to the transition frequencies (i.e., the differences between the energies of the consecutive energy levels) are similar.

An important issue that needs to be discussed is the accuracy and the level of convergence of our calculations. To provide an answer to this point we have performed an analysis of the convergence of the total nonrelativistic energies for all states using an approximate extrapolation procedure. The results of the extrapolation are shown in Table II in the column marked $\Delta E^{\text {nrel }}$. The value shown there for each state is the energy difference between the energy obtained with 5800 basis functions and the energy value extrapolated to the infinite number of basis functions. As one can see, $\Delta E^{\text {nrel }}$ increases from $2 \times 10^{-10}$ hartree for the lowest state to 5 $\times 10^{-9}$ hartree for the highest indicating that, as expected, the lower states are converged tighter than the higher ones.
However, for all states the convergence is quite satisfactory particularly for calculating transition energies, which are energy differences between the adjacent states. We also performed an analysis of the absolute error in determining the total $\alpha^{2}$ relativistic corrections. For the analysis we used the results obtained for the lowest $(v=0)$, the highest $(v=14)$, and an intermediate vibrational state $(v=8)$ with the number of basis functions equal to 5200, 5400, 5600, 5800, and 6000. The analysis is presented in Table IV. As one can see the estimated error for all three states does not exceed 3 $\times 10^{-10}$ hartree. Thus, the precision the calculation of the relativistic corrections can be deemed as quite satisfactory.

The transition frequencies obtained in the calculations are presented in Table V. We show the results obtained with and without the relativistic corrections (the first two columns in the table). The frequencies are compared with the frequencies obtained by Wolniewicz. ${ }^{34,35}$ There are two columns in Table V with Wolniewicz's results. In the first column the frequencies were obtained from energies that include the $\alpha^{2}$ relativistic corrections. The frequencies in the second column also include radiative corrections estimated with an approximate method. We should mention that there is a difference between our approach and the way Wolniewicz performed his calculations. While, as described above, no distinction is made in the way the particles forming the molecule are treated in our approach, the approach used by Wolniewicz was based on the Born-Oppenheimer approximation that produced the zero-order wave function which was subsequently used to calculate finite-mass corrections and the relativistic corrections. The potential energy curve, which includes those corrections, is then used to determine the vibrational states. Table $\mathrm{V}$ also includes a comparison with the experimental frequencies.

Let us first discuss the comparison of our results with the results of Wolniewicz. In general, the agreement between our frequencies obtained with relativistic corrections is very good. The differences are not more than a few thousands of a wave number. The largest differences appear for the highest two states where they approach $0.01 \mathrm{~cm}^{-1}$. The agreement between the $\mathrm{BO}$ and non-BO results indicates that the newly developed algorithm for calculating the orbit-orbit interaction works correctly. The small differences between the $\alpha^{2}$ relativistic corrections obtained with the two approaches may become important when the theory and the experiment will improve and reach the accuracy of $0.01 \mathrm{~cm}^{-1}$. This particularly concerns the higher excited states.

Lastly, let us focus on the comparison of the calculated frequencies with the experimental values. Apart from the first transition, for which a new estimate was generated in the

TABLE IV. Convergence of the total relativistic $\alpha^{2}$ correction for selected states and an estimate of its absolute error. All quantities are in hartrees.

\begin{tabular}{rcccccc}
\hline \hline$v$ & $\begin{array}{c}5200 \\
\left(\times 10^{-5}\right)\end{array}$ & $\begin{array}{c}5400 \\
\left(\times 10^{-5}\right)\end{array}$ & $\begin{array}{c}5600 \\
\left(\times 10^{-5}\right)\end{array}$ & $\begin{array}{c}5800 \\
\left(\times 10^{-5}\right)\end{array}$ & $\begin{array}{c}6000 \\
\left(\times 10^{-5}\right)\end{array}$ & $\begin{array}{c}\text { est error } \\
\left(\times 10^{-10}\right)\end{array}$ \\
\hline 0 & -1.066792 & -1.066794 & -1.066816 & -1.066815 & -1.066812 & 1 \\
8 & -1.064414 & -1.064393 & -1.064443 & -1.064452 & -1.064449 & 3 \\
14 & -1.266762 & -1.266803 & -1.266790 & -1.266817 & -1.266829 & 3 \\
\hline \hline
\end{tabular}


TABLE V. Pure vibrational transition frequencies for $\mathrm{H}_{2}$ calculated using the nonrelativistic $\left(E_{v+1}^{\mathrm{nr}}-E_{v}^{\mathrm{nr}}\right)$ and relativistic $\left(E_{v+1}^{\mathrm{rel}}-E_{v}^{\mathrm{rel}}\right)$ total energies. Our transition frequencies are compared with the frequencies obtained by Wolniewicz using his total energies corrected for $\alpha^{2}$ relativistic effects (Wolniewicz 1) and for in addition for radiative effects (Wolniewicz 2).

\begin{tabular}{lrrccc}
\hline \hline$v$ & $E_{v+1}^{\mathrm{nr}}-E_{v}^{\mathrm{nr}}$ & $E_{v+1}^{\mathrm{rel}}-E_{v}^{\mathrm{rel}}$ & Wolniewicz 1 35 and 36 & Wolniewicz 2 35 and 36 & Experiment 22 \\
\hline $1 \rightarrow 0$ & 4161.16416 & 4161.1856 & 4161.1863 & 4161.167 & $4161.1660(3)$ \\
$2 \rightarrow 1$ & 3925.83864 & 3925.8553 & 3925.8549 & 3925.836 & 3925.79 \\
$3 \rightarrow 2$ & 3695.39468 & 3695.4059 & 3695.4042 & 3695.389 & 3695.43 \\
$4 \rightarrow 3$. & 3467.98592 & 3467.9910 & 3467.9894 & 3467.976 & 3467.95 \\
$5 \rightarrow 4$ & 3241.58052 & 3241.5793 & 3241.5770 & 3241.564 & 3241.61 \\
$6 \rightarrow 5$ & 3013.87201 & 3013.8640 & 3013.8622 & 3013.851 & 3013.86 \\
$7 \rightarrow 6$ & 2782.16474 & 2782.1488 & 2782.1466 & 2782.136 & 2782.13 \\
$8 \rightarrow 7$ & 2543.21194 & 2543.1879 & 2543.1859 & 2543.175 & 2543.25 \\
$9 \rightarrow 8$ & 2292.99557 & 2292.9613 & 2292.9601 & 2292.950 & 229.93 \\
$10 \rightarrow 9$ & 2026.40685 & 2026.3611 & 2026.3615 & 2026.351 & 2026.38 \\
$11 \rightarrow 10$ & 1736.77644 & 1736.7167 & 1736.7174 & 1736.707 & 1736.66 \\
$12 \rightarrow 11$ & 1415.16098 & 1415.0840 & 1415.0872 & 1415.076 & 1415.07 \\
$13 \rightarrow 12$ & 1049.24570 & 1049.1461 & 1049.1522 & 1049.139 & 1049.16 \\
$14 \rightarrow 13$ & 622.09089 & 621.9622 & 621.9720 & 621.956 & 622.02 \\
\hline \hline
\end{tabular}

present work with the error bar of about $\pm 0.002 \mathrm{~cm}^{-1}$, the other transitions have the error bar of about $0.1 \mathrm{~cm}^{-1}$. Since the difference between the calculated and the experimental transitions is less than that, it is difficult to tell how precise the calculations are. Thus, the only hint regarding the accuracy of the calculation has to come from the comparison of the results for the lowest $(1 \rightarrow 0)$ transition. Our result for this transition of $4161.1862 \mathrm{~cm}^{-1}$ agrees perfectly with the transition of $4161.1863 \mathrm{~cm}^{-1}$ obtained by Wolniewicz. However, both are off from the experimental transition of $4161.1660(3) \mathrm{cm}^{-1}$ by more than $0.02 \mathrm{~cm}^{-1}$. An approximate treatment of the radiative effects by Wolniewicz lowers his calculated transition to $4161.167 \mathrm{~cm}^{-1}$. This change is in the right direction, and only recovers most of the $0.03 \mathrm{~cm}^{-1}$ difference. It is possible that a more precise calculation of the radiative effects would further reduce the difference between the theory and the experiment. Such calculations will need to include QED effects and effects due to the finite structure of the nuclei. With that an agreement within the experimental error bar of $0.002 \mathrm{~cm}^{-1}$ for the $1 \rightarrow 0$ transition may be achievable with the calculations.

\section{SUMMARY}

In conclusion, the results presented in this work demonstrate that the approach based on using the high-accuracy non-BO wave function in evaluating the $\alpha^{2}$ relativistic correction yields results that match the high-resolution experimental transition energies with the accuracy approaching a few hundreds of a wave number. To further increase the accuracy of the calculations one needs to include the lowestorder QED corrections, as well as corrections due to the finite size of the nuclei and their polarizabilities. Our future effort will go in this direction.

With the complete $\alpha^{2}$ relativistic correction now implemented within the non-BO framework for diatomic molecules with $\sigma$ electrons, we plan to perform some more application calculations. Apart from two electron systems such as isotopomers of $\mathrm{H}_{2}$ or $\mathrm{HeH}^{+}$, we are particularly interested in three- and four-electron molecules. Our approach is the only one developed so far that allows studying such systems without the BO approximation and with very high accuracy. We hope that calculations performed at such an accuracy level will inspire new high-resolution experimental work aiming at measuring the transitions that we consider in the calculations. Clearly such work is needed for the $\mathrm{H}_{2}$ molecule where only the lowest pure vibrational transition is known with the precision higher than that of the calculations.

\section{ACKNOWLEDGMENTS}

This work has been supported in part by the National Science Foundation. We are grateful to the University of Arizona Center of Computing and Information Technology for using their supercomputer resources.

\section{APPENDIX A: THE EXPECTATION VALUE OF THE ORBIT-ORBIT INTERACTION OPERATOR}

As in our previous works, also here we use a matrix approach to evaluate the integrals that appear in the expectation value of the orbit-orbit interaction Hamiltonian, $\hat{H}_{O O}(\mathbf{r})$. This approach has been shown to greatly simplify the derivations of the matrix elements and to lead to compact expressions for the integrals. In the derivations below we use $3 n \times 3 n$ matrices where $n$ is the number of pseudoparticles in the system. The matrix formalism used here was described in Ref. 36.

The expectation value of the orbit-orbit operator are derived for explicitly correlated Gaussian basis functions in the form

$$
\phi=r_{1}^{m} \exp \left[-\mathbf{r}^{\prime} \overline{\mathbf{A}} \mathbf{r}\right],
$$

where

$$
\overline{\mathbf{A}}=\mathbf{A} \otimes \mathbf{1}_{3},
$$

and $\mathbf{A}$ is a $n \times n$ symmetric matrix of the exponential coefficients, $\mathbf{1}_{3}$ is a $3 \times 3$ unit matrix, and $\otimes$ is a Kronecker 
matrix product, and the $m$ powers of $r_{1}$ are non-negative even numbers.

\section{Some auxiliary formulas}

There are some simple relations that have used in deriving the expressions for the integrals. They are

- $\mathbf{r}_{i}^{2}=\mathbf{r}^{\prime} \overline{\mathbf{J}}_{i i} \mathbf{r}$

- $\mathbf{r}_{i j}=\mathbf{r}_{i}-\mathbf{r}_{j}$;

- because of $\overline{\mathbf{A}}=\mathbf{A} \otimes \mathbf{I}_{3}$ we have the following relations:

$\operatorname{tr}[\overline{\mathbf{A}}]=3 \operatorname{tr}[\mathbf{A}]$,

$|\overline{\mathbf{A}}|=|\mathbf{A}|^{3}$.

- By $\nabla_{\mathbf{r}}$ we denote the gradient with respect to the vector of the pseudoparticle coordinates $\mathbf{r}$, and we have ( $\phi$ $\left.=r_{1}^{m} \exp \left[-\mathbf{r}^{\prime} \overline{\mathbf{A}} \mathbf{r}\right]\right)$ :

$\nabla_{\mathbf{r}}^{\alpha} \phi \equiv \nabla^{\alpha} \phi \quad$ and $\nabla_{\mathbf{r} \alpha} \phi \equiv \nabla_{\alpha} \phi$.

- In this work we use the following first-order derivatives:

$$
\begin{aligned}
& \nabla^{\alpha} \phi=\left(m r_{1}^{-2}\left(\mathbf{r}^{\prime} \overline{\mathbf{J}}_{11}\right)^{\alpha}-2\left(\mathbf{r}^{\prime} \overline{\mathbf{A}}\right)^{\alpha}\right) \phi \\
& \nabla_{\alpha} \phi=\left(m r_{1}^{-2}\left(\overline{\mathbf{J}}_{11} \mathbf{r}\right)_{\alpha}-2(\overline{\mathbf{A}} \mathbf{r})_{\alpha}\right) \phi
\end{aligned}
$$

where

$$
\begin{aligned}
& \nabla^{\alpha}\left(\mathbf{r}^{\prime} \overline{\mathbf{A}} \mathbf{r}\right)=2\left(\mathbf{r}^{\prime} \overline{\mathbf{A}}\right)^{\alpha}, \\
& \nabla_{\alpha}\left(\mathbf{r}^{\prime} \overline{\mathbf{A}} \mathbf{r}\right)=2(\overline{\mathbf{A}} \mathbf{r})_{\alpha}, \\
& \nabla^{\alpha} r_{1}^{m}=m r_{1}^{m} r_{1}^{-2}\left(\mathbf{r}^{\prime} \overline{\mathbf{J}}_{11}\right)^{\alpha}, \\
& \nabla_{\alpha} r_{1}^{m}=m r_{1}^{m} r_{1}^{-2}\left(\overline{\mathbf{J}}_{11} \mathbf{r}\right)_{\alpha}, \\
& \nabla_{j}^{\beta} r_{i j}^{\alpha}=-\nabla_{j}^{\beta} r_{j}^{\alpha}=-\delta^{\beta \alpha}
\end{aligned}
$$

- and the following second-order derivatives:

$$
\begin{aligned}
\nabla_{\beta} \nabla_{\alpha} \phi= & r_{1}^{-4} m(m-2)\left(\overline{\mathbf{J}}_{11} \mathbf{r}\right)_{\alpha}\left(\overline{\mathbf{J}}_{11} \mathbf{r}\right)_{\beta} \phi \\
& -2 r_{1}^{-2} m\left[\left(\mathbf{J}_{11} \mathbf{r}\right)_{\alpha}(\overline{\mathbf{A}} \mathbf{r})_{\beta}+(\overline{\mathbf{A}} \mathbf{r})_{\alpha}\left(\overline{\mathbf{J}}_{11} \mathbf{r}\right)_{\beta}\right] \phi \\
& +r_{1}^{-2} m\left(\overline{\mathbf{J}}_{11}\right)_{\alpha \beta} \phi+4(\overline{\mathbf{A}} \mathbf{r})_{\alpha}(\overline{\mathbf{A}} \mathbf{r})_{\beta} \phi-2 \overline{\mathbf{A}}_{\alpha \beta} \phi,
\end{aligned}
$$

$$
\begin{aligned}
\nabla^{\beta} \nabla_{\alpha} \phi= & r_{1}^{-4} m(m-2)\left(\mathbf{r}^{\prime} \overline{\mathbf{J}}_{11}\right)^{\beta}\left(\overline{\mathbf{J}}_{11} \mathbf{r}\right)_{\alpha} \phi \\
& -2 r_{1}^{-2} m\left[\left(\mathbf{r}^{\prime} \overline{\mathbf{A}}\right)^{\beta}\left(\overline{\mathbf{J}}_{11} \mathbf{r}\right)_{\alpha}+\left(\mathbf{r}^{\prime} \overline{\mathbf{J}}_{11}\right)^{\beta}(\overline{\mathbf{A}} \mathbf{r})_{\alpha}\right] \phi \\
& +r_{1}^{-2} m\left(\overline{\mathbf{J}}_{11}\right)_{\alpha}^{\beta} \phi+4\left(\mathbf{r}^{\prime} \overline{\mathbf{A}}\right)^{\beta}(\overline{\mathbf{A}} \mathbf{r})_{\alpha} \phi-2 \overline{\mathbf{A}}_{\alpha}^{\beta} \phi .
\end{aligned}
$$

- We define the matrix $\mathbf{J}_{i j}$ used in the above expressions as
$\mathbf{J}_{i j}=\left\{\begin{array}{cc}\mathbf{E}_{i i} & i=j \\ \mathbf{E}_{i i}+\mathbf{E}_{j j}-\mathbf{E}_{i j}-\mathbf{E}_{j i} & i \neq j,\end{array}\right\}$,

where $\mathbf{E}_{i j}$ is the $n \times n$ matrix with 1 in the $i j$ th position and 0's elsewhere. Formally this can be written as $\left(\mathbf{E}_{i j}\right)_{\beta}^{\alpha}=\delta_{i}^{\alpha} \delta_{j \beta}$. It is easy to see that

$\mathbf{E}_{i j} \cdot \mathbf{E}_{i j}=0$,

$\mathbf{E}_{i j} \cdot \mathbf{E}_{j i}=\mathbf{E}_{i i}$,

$\mathbf{E}_{i j} \cdot \mathbf{E}_{j j}=\mathbf{E}_{i j}$

- With the above-defined quantities it can be now shown that the operators $\nabla_{i} \cdot \nabla_{j}$, and $\mathbf{r}_{i j}^{\prime} \nabla_{j}$ can be expressed as

$$
\begin{aligned}
\nabla_{i} \cdot \nabla_{j} & =\nabla^{\prime} \overline{\mathbf{E}}_{i j} \nabla, \\
\mathbf{r}_{i j} \cdot \nabla_{j} & =\left(\mathbf{r}_{i}-\mathbf{r}_{j}\right) \cdot \nabla_{j} \\
& =\mathbf{r}^{\prime}\left(\overline{\mathbf{E}}_{i j}-\overline{\mathbf{E}}_{j j}\right) \nabla \\
& =\mathbf{r}^{\prime}\left(\overline{\mathbf{E}}_{i j}-\overline{\mathbf{E}}_{j j}\right) \overline{\mathbf{E}}_{j j} \nabla,
\end{aligned}
$$

where $\mathbf{r}_{i j}=\mathbf{r}_{i}-\mathbf{r}_{j}$.

Finally, to reduce the singularity of the orbit-orbit operator, which appears due to the term $1 / r_{i j}^{3}$, we employ the following commutation relations:

$$
\begin{aligned}
& {\left[\nabla_{i}, \frac{1}{r_{i}}\right]=-\frac{\mathbf{r}_{i}}{r_{i}^{3}},} \\
& {\left[\nabla_{j}, \frac{1}{r_{i j}}\right]=-\left[\nabla_{i}, \frac{1}{r_{i j}}\right]=\frac{\mathbf{r}_{i j}}{r_{i j}^{3}},}
\end{aligned}
$$

\section{The orbit-orbit interaction operator}

In the internal coordinate system the orbit-orbit operator has the following form:

$$
\begin{aligned}
\hat{H}_{O O}(r)= & -\frac{1}{2} \sum_{i=i}^{n} \frac{q_{0} q_{i}}{m_{0} m_{i}}\left\{\frac{1}{r_{i}} \nabla_{i} \cdot \nabla_{i}+\frac{1}{r_{i}^{3}} \mathbf{r}_{i} \cdot\left(\mathbf{r}_{i} \cdot \nabla_{i}\right) \nabla_{i}\right\} \\
& -\frac{1}{2} \sum_{i=1}^{n} \sum_{j \neq i}^{n} \frac{q_{0} q_{i}}{m_{0} m_{i}}\left\{\frac{1}{r_{i}} \nabla_{i} \cdot \nabla_{j}+\frac{1}{r_{i}^{3}} r_{i} \cdot\left(\mathbf{r}_{i} \cdot \nabla_{i}\right) \nabla_{j}\right\} \\
& +\frac{1}{2} \sum_{i=1}^{n-1} \sum_{j>i}^{n} \frac{q_{i} q_{j}}{m_{i} m_{j}}\left\{\frac{1}{r_{i j}} \nabla_{i} \cdot \nabla_{j}\right. \\
& \left.+\frac{1}{r_{i j}^{3}} \mathbf{r}_{i j} \cdot\left(\mathbf{r}_{i j} \cdot \nabla_{i}\right) \nabla_{j}\right\} .
\end{aligned}
$$

Using the relations (15) and (16) the matrix notation described in our previous work $^{37}$ the expectation value of the orbit-orbit interaction operator is 


$$
\begin{aligned}
\left\langle\phi_{K}\left|\hat{H}_{O O}(\mathbf{r})\right| \phi_{L}\right\rangle & \\
= & -\frac{1}{2} \sum_{i=1}^{n} \frac{q_{0} q_{i}}{m_{0} m_{i}}\left\langle\phi_{K}\right| \frac{1}{r_{i}} \nabla^{\prime} \overline{\mathbf{E}}_{i i} \nabla \\
& -\left(\mathbf{r}^{\prime} \overline{\mathbf{E}}_{i i}\right)^{\alpha}\left(\nabla^{\prime} \overline{\mathbf{E}}_{i i} \frac{1}{r_{i}}\right)^{\beta}\left(\overline{\mathbf{E}}_{i i} \nabla\right)_{\beta}\left(\overline{\mathbf{E}}_{i i} \nabla\right)_{\alpha}\left|\phi_{L}\right\rangle \quad(\mathrm{A} 7) \\
& -\frac{1}{2} \sum_{i=1}^{n} \sum_{j \neq i}^{n} \frac{q_{0} q_{i}}{m_{0} m_{i}}\left\langle\phi_{K}\right| \frac{1}{r_{i}} \nabla^{\prime} \overline{\mathbf{E}}_{i j} \nabla \\
& -\left(\mathbf{r}^{\prime} \overline{\mathbf{E}}_{i i}\right)^{\alpha}\left(\nabla^{\prime} \overline{\mathbf{E}}_{i i} \frac{1}{r_{i}}\right)^{\beta}\left(\overline{\mathbf{E}}_{i i} \nabla\right)_{\beta}\left(\overline{\mathbf{E}}_{i j} \nabla\right)_{\alpha}\left|\phi_{L}\right\rangle \\
& +\frac{1}{2} \sum_{i=1}^{n-1} \sum_{j>i}^{n} \frac{q_{i} q_{j}}{m_{i} m_{j}}\left\langle\phi_{K}\right| \frac{1}{r_{i j}} \nabla^{\prime} \overline{\mathbf{E}}_{i j} \nabla+\left(\mathbf{r}^{\prime}\left(\overline{\mathbf{E}}_{i j}-\mathbf{E}_{j j}\right)\right)^{\alpha} \\
& \times\left(\nabla^{\prime} \overline{\mathbf{E}}_{j i} \frac{1}{r_{i j}}\right)^{\beta}\left(\overline{\mathbf{E}}_{i i} \nabla\right)_{\beta}\left(\overline{\mathbf{E}}_{j j} \nabla\right)_{\alpha}\left|\phi_{L}\right\rangle .
\end{aligned}
$$

To simplify this expression we use the following general integral for each of the three terms that appear in the expectation value:

$$
\begin{aligned}
& \left\langle\phi_{K}\left|\frac{1}{r_{g}} \nabla^{\prime} \overline{\mathbf{B}} \nabla\right| \phi_{L}\right\rangle \\
& \quad-\left\langle\phi_{K}\left|\left(\mathbf{r}^{\prime} \overline{\mathbf{C}}\right)^{\alpha}\left(\nabla^{\prime} \overline{\mathbf{D}} \frac{1}{r_{g}}\right)^{\beta}(\overline{\mathbf{F}} \nabla)_{\beta}(\overline{\mathbf{G}} \nabla)_{\alpha}\right| \phi_{L}\right\rangle
\end{aligned}
$$

for term (17): $g=i \overline{\mathbf{B}}=\overline{\mathbf{E}}_{i i} \quad \overline{\mathbf{C}}=\overline{\mathbf{E}}_{i i} \quad \overline{\mathbf{D}}=\overline{\mathbf{E}}_{i i} \quad \overline{\mathbf{F}}=\overline{\mathbf{E}}_{i i}$

$\overline{\mathbf{G}}=\overline{\mathbf{E}}_{i i}$,

for term (18): $g=i \overline{\mathbf{B}}=\overline{\mathbf{E}}_{i j} \quad \overline{\mathbf{C}}=\overline{\mathbf{E}}_{i i} \quad \overline{\mathbf{D}}=\overline{\mathbf{E}}_{i i}$

$$
\overline{\mathbf{F}}=\overline{\mathbf{E}}_{i i} \quad \overline{\mathbf{G}}=\overline{\mathbf{E}}_{i j},
$$$$
\text { for term (19): } g=i j \quad \overline{\mathbf{B}}=\overline{\mathbf{E}}_{i j} \quad \overline{\mathbf{C}}=\left(\overline{\mathbf{E}}_{i j}-\overline{\mathbf{E}}_{j j}\right)
$$

$$
\overline{\mathbf{D}}=\overline{\mathbf{E}}_{j i} \quad \overline{\mathbf{F}}=\overline{\mathbf{E}}_{i i} \quad \overline{\mathbf{G}}=\overline{\mathbf{E}}_{j j} .
$$

Hence, in order to calculate the expectation value of $\hat{H}_{o o}(\mathbf{r})$ we need to evaluate the following integrals:

$$
\left\langle\phi_{K}\left|\frac{1}{r_{g}} \nabla^{\prime} \overline{\mathbf{B}} \nabla\right| \phi_{L}\right\rangle
$$

and

$$
\left\langle\phi_{K}\left|\left(\mathbf{r}^{\prime} \overline{\mathbf{C}}\right)^{\alpha}\left(\nabla^{\prime} \overline{\mathbf{D}} \frac{1}{r_{g}}\right)^{\beta}(\overline{\mathbf{F}} \nabla)_{\beta}(\overline{\mathbf{G}} \nabla)_{\alpha}\right| \phi_{L}\right\rangle .
$$

We now derive the formulas for these two integrals.

\section{Integral $\left\langle\phi_{K}\left|1 / r_{g} \nabla^{\prime} \bar{B} \nabla\right| \phi_{L}\right\rangle$}

First we apply the operator $\nabla^{\prime} \overline{\mathbf{B}} \nabla$ to $\phi_{L}$ and using relation (15) we get

$$
\begin{aligned}
\nabla^{\prime} \overline{\mathbf{B}} \nabla \phi_{L}= & \nabla^{\beta}(\overline{\mathbf{B}})_{\beta}^{\alpha} \nabla_{\alpha} \phi_{L}=\overline{\mathbf{B}}_{\beta}^{\alpha} \nabla^{\beta} \phi_{L} \\
\overline{\mathbf{B}}_{\beta}^{\alpha} \nabla^{\beta} \nabla_{\alpha} \phi_{L}= & r_{1}^{-4} m_{L}\left(m_{L}-2\right)\left(\mathbf{r}^{\prime} \overline{\mathbf{J}}_{11}\right)^{\beta} \overline{\mathbf{B}}_{\beta}^{\alpha}\left(\overline{\mathbf{J}}_{11} \mathbf{r}\right)_{\alpha} \phi_{L} \\
& -2 r_{1}^{-2} m_{L}\left[\left(\mathbf{r}^{\prime} \overline{\mathbf{A}}_{L}\right)_{\beta} \overline{\mathbf{B}}_{\beta}^{\alpha}\left(\overline{\mathbf{J}}_{11} \mathbf{r}\right)_{\alpha}\right. \\
& \left.+\left(\mathbf{r}^{\prime} \overline{\mathbf{J}}_{11}\right)_{\beta} \overline{\mathbf{B}}_{\beta}^{\alpha}\left(\overline{\mathbf{A}}_{L} \mathbf{r}\right)_{\alpha}\right] \phi_{L} \\
& +r_{1}^{-2} m_{L} \overline{\mathbf{B}}_{\beta}^{\alpha}\left(\overline{\mathbf{J}}_{11}\right)_{\alpha \beta} \phi_{L} \\
& +4\left(\mathbf{r}^{\prime} \overline{\mathbf{A}}_{L}\right)_{\beta} \overline{\mathbf{B}}_{\beta}^{\alpha}\left(\overline{\mathbf{A}}_{L} \mathbf{r}\right)_{\alpha} \phi_{L}-2\left(\overline{\mathbf{A}}_{L}\right)_{\alpha \beta} \overline{\mathbf{B}}_{\beta}^{\alpha} \phi_{L} .
\end{aligned}
$$

With that we can now calculate the value of the integral

$$
\begin{aligned}
\left\langle\phi_{K}\left|\frac{1}{r_{g}} \nabla^{\prime} \overline{\mathbf{B}} \nabla\right| \phi_{L}\right\rangle & =m_{L}\left(m_{L}-2\right)\left\langle\phi_{K}\left|\frac{1}{r_{g}} r_{1}^{-4}\left(\mathbf{r}^{\prime} \overline{\mathbf{J}}_{11} \overline{\mathbf{B}} \overline{\mathbf{J}}_{11} \mathbf{r}\right)\right| \phi_{L}\right\rangle \\
& -2 m_{L}\left\langle\phi_{K}\left|\frac{1}{r_{g}} r_{1}^{-2}\left[\left(\mathbf{r}^{\prime} \overline{\mathbf{A}}_{L} \overline{\mathbf{B}}_{11} \mathbf{r}\right)+\left(\mathbf{r}^{\prime} \overline{\mathbf{J}}_{11} \overline{\mathbf{B}} \overline{\mathbf{A}}_{L} \mathbf{r}\right)\right]\right| \phi_{L}\right\rangle \\
& +3 m_{L} \operatorname{tr}\left[\mathbf{B} \mathbf{J}_{11}\right]\left\langle\phi_{K}\left|\frac{1}{r_{g}} r_{1}^{-2}\right| \phi_{L}\right\rangle \\
& +4\left\langle\phi_{K}\left|\frac{1}{r_{g}}\left(\mathbf{r}^{\prime} \overline{\mathbf{A}}_{L} \overline{\mathbf{B}}_{\overline{\mathbf{A}}} \overline{\mathbf{A}}_{L} \mathbf{r}\right)\right| \phi_{L}\right\rangle-6 \operatorname{tr}\left[\mathbf{A}_{L} \mathbf{B}\right] \\
& \times\left\langle\phi_{K}\left|\frac{1}{r_{g}}\right| \phi_{L}\right\rangle .
\end{aligned}
$$

The integrals that appear in the above expression were either derived in our previous work ${ }^{16}$ or are derived in Appendix B in this work.

\section{Integral $\left\langle\phi_{K}\left|\left(\mathbf{r}^{\prime} \overline{\mathbf{C}}\right)^{\alpha}\left(\nabla^{\prime} \bar{D} 1 / r_{g}\right)^{\beta}(\overline{\mathbf{F}} \nabla)_{\beta}(\overline{\mathbf{G}} \nabla)_{\alpha}\right| \phi_{L}\right\rangle$}

We rewrite this integral in the following way:

$$
\begin{aligned}
& \left\langle\phi_{K}\left|\left(\mathbf{r}^{\prime} \overline{\mathbf{C}}\right)^{\alpha}\left(\nabla^{\prime} \overline{\mathbf{D}} \frac{1}{r_{g}}\right)^{\beta}(\overline{\mathbf{F}} \nabla)_{\beta}(\overline{\mathbf{G}} \nabla)_{\alpha}\right| \phi_{L}\right\rangle \\
& =-\int d \tau \frac{1}{r_{g}}\left(\nabla^{\prime} \overline{\mathbf{D}}\right)^{\beta}\left\{\phi_{K}\left(\mathbf{r}^{\prime} \overline{\mathbf{C}}\right)^{\alpha}(\overline{\mathbf{F}} \nabla)_{\beta}(\overline{\mathbf{G}} \nabla)_{\alpha} \phi_{L}\right\},
\end{aligned}
$$

where the operator involved in the integral can be split into three terms

$$
\begin{aligned}
\left(\nabla^{\prime} \overline{\mathbf{D}}\right)^{\beta} & \left\{\phi_{K}\left(\mathbf{r}^{\prime} \overline{\mathbf{C}}\right)^{\alpha}(\overline{\mathbf{F}} \nabla)_{\beta}(\overline{\mathbf{G}} \nabla)_{\alpha} \phi_{L}\right\} \\
= & \left\{\left(\nabla^{\prime} \overline{\mathbf{D}}\right)^{\beta} \phi_{K}\right\}\left(\mathbf{r}^{\prime} \overline{\mathbf{C}} \overline{\mathbf{G}} \nabla\right)(\overline{\mathbf{F}} \nabla)_{\beta} \phi_{L} \equiv \text { term } 1 \\
& +\phi_{K}\left\{\left(\nabla^{\prime} \overline{\mathbf{D}}\right)^{\beta}\left(\mathbf{r}^{\prime} \overline{\mathbf{C}}\right)^{\alpha}\right\}(\overline{\mathbf{F}} \nabla)_{\beta}(\overline{\mathbf{G}} \nabla)_{\alpha} \phi_{L}=\text { term } 2 \\
& +\phi_{K}\left(\mathbf{r}^{\prime} \overline{\mathbf{C}}\right)^{\alpha}\left\{\left(\nabla^{\prime} \overline{\mathbf{D}}\right)^{\beta}(\overline{\mathbf{F}} \nabla)_{\beta}(\overline{\mathbf{G}} \nabla)_{\alpha} \phi_{L}\right\}, \equiv \text { term } 3,
\end{aligned}
$$

Now we evaluate each term separately. 
(1) $\quad$ Term $1\left\{\left(\nabla^{\prime} \overline{\mathbf{D}}\right)^{\beta} \phi_{K}\right\}\left(\mathbf{r}^{\prime} \overline{\mathbf{C}} \overline{\mathbf{G}} \nabla\right)(\overline{\mathbf{F}} \nabla)_{\beta} \phi_{L}$

$\left(\nabla^{\prime} \overline{\mathbf{D}}\right)^{\beta} \phi_{K}=\overline{\mathbf{D}}_{\gamma}^{\beta} \nabla^{\gamma} \phi_{K}$

$$
=\left[m_{K} r_{1}^{-2}\left(\mathbf{r}^{\prime} \overline{\mathbf{J}}_{11} \overline{\mathbf{D}}\right)^{\beta}-2\left(\mathbf{r}^{\prime} \overline{\mathbf{A}}_{K} \overline{\mathbf{D}}\right)^{\beta}\right] \phi_{K},
$$

$(\mathbf{r}, \overline{\mathbf{C}} \overline{\mathbf{G}} \nabla)(\overline{\mathbf{F}} \nabla)_{\beta}^{\prime} \boldsymbol{\phi}_{L}$

$$
\begin{aligned}
= & \left(\mathbf{r}^{\prime} \overline{\mathbf{C}} \overline{\mathbf{G}}\right)^{\alpha} \overline{\mathbf{F}}_{\alpha}^{\rho} \nabla_{\alpha} \nabla_{\rho} \phi_{L} \\
= & r_{1}^{-4} m_{L}\left(m_{L}-2\right)\left(\mathbf{r}^{\prime} \overline{\mathbf{C}} \overline{\mathbf{G}} \overline{\mathbf{J}}_{11} \mathbf{r}\right)\left(\overline{\mathbf{F}} \overline{\mathbf{J}}_{11} \mathbf{r}\right)_{\beta} \phi_{L} \\
& +r_{1}^{-2} m_{L}\left(\mathbf{r}^{\prime} \overline{\mathbf{C}} \overline{\mathbf{G}} \overline{\mathbf{J}}_{11} \overline{\mathbf{F}}^{\prime}\right)_{\beta} \phi_{L}-2 r_{1}^{-2} m_{L}
\end{aligned}
$$

$$
\begin{aligned}
& \int d \tau\left\{\left(\nabla^{\prime} \overline{\mathbf{D}}\right)^{\beta} \phi_{K}\right\}\left(\mathbf{r}^{\prime} \overline{\mathbf{C}} \overline{\mathbf{G}} \nabla\right)(\overline{\mathbf{F}} \nabla)_{\beta} \phi_{L} \\
& =m_{K} m_{L}\left(m_{L}-2\right)\left\langle\phi_{K}\left|\frac{1}{r_{g}} r_{1}^{-6}\left(\mathbf{r}^{\prime} \overline{\mathbf{C}} \overline{\mathbf{G}} \overline{\mathbf{J}}_{11} \mathbf{r}\right)\left(\mathbf{r}^{\prime} \overline{\mathbf{J}}_{11} \overline{\mathbf{D}} \overline{\mathbf{F}} \overline{\mathbf{J}}_{11} \mathbf{r}\right)\right| \phi_{L}\right\rangle-2 m_{L}\left(m_{L}-2\right)\left\langle\phi_{K}\left|\frac{1}{r_{g}} r_{1}^{-4}\left(\mathbf{r}^{\prime} \overline{\mathbf{C}} \overline{\mathbf{G}} \overline{\mathbf{J}}_{11} \mathbf{r}\right)\left(\mathbf{r}^{\prime} \overline{\mathbf{A}}_{K} \overline{\mathbf{D}} \overline{\mathbf{F}} \overline{\mathbf{J}}_{11} \mathbf{r}\right)\right| \phi_{L}\right\rangle
\end{aligned}
$$

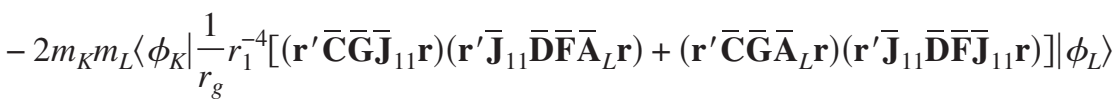

$$
\begin{aligned}
& +m_{K} m_{L}\left\langle\phi_{K}\left|\frac{1}{r_{g}} r_{1}^{-4}\left(\mathbf{r}^{\prime} \overline{\mathbf{J}}_{11} \overline{\mathbf{D}} \overline{\mathbf{F}} \overline{\mathbf{J}}_{11} \overline{\mathbf{G}}^{\prime} \overline{\mathbf{C}}^{\prime} \mathbf{r}\right)\right| \phi_{L}\right\rangle+4 m_{L}\left\langle\phi_{K}\right| \frac{1}{r_{g}} r_{1}^{-2}\left[\left(\mathbf{r}^{\prime} \overline{\mathbf{C}} \overline{\mathbf{G}} \overline{\mathbf{J}}_{11} \mathbf{r}\right)\left(\mathbf{r}^{\prime} \overline{\mathbf{A}}_{K} \overline{\mathbf{D}} \overline{\mathbf{F}} \overline{\mathbf{A}}_{L} \mathbf{r}\right)\right. \\
& \left.+\left(\mathbf{r}^{\prime} \overline{\mathbf{C}} \overline{\mathbf{G}}_{\overline{\mathbf{A}}_{L}} \mathbf{r}\right)\left(\mathbf{r}^{\prime} \overline{\mathbf{A}}_{K} \overline{\mathbf{D}} \overline{\mathbf{F}} \overline{\mathbf{J}}_{11} \mathbf{r}\right)\right]\left|\phi_{L}\right\rangle-2 m_{L}\left\langle\phi_{K}\left|\frac{1}{r_{g}} r_{1}^{-2}\left(\mathbf{r}^{\prime} \overline{\mathbf{A}}_{K} \overline{\mathbf{D}} \overline{\mathbf{F}}_{11} \overline{\mathbf{G}}^{\prime} \overline{\mathbf{C}}^{\prime} \mathbf{r}\right)\right| \phi_{L}\right\rangle \\
& +4 m_{K}\left\langle\phi_{K}\left|\frac{1}{r_{g}} r_{1}^{-2}\left(\mathbf{r}^{\prime} \overline{\mathbf{C}} \overline{\mathbf{G}} \overline{\mathbf{A}}_{L} \mathbf{r}\right)\left(\mathbf{r}^{\prime} \overline{\mathbf{J}}_{11} \overline{\mathbf{D}} \mathbf{F} \overline{\mathbf{A}}_{L} \mathbf{r}\right)\right| \phi_{L}\right\rangle \\
& -2 m_{K}\left\langle\phi_{K}\left|\frac{1}{r_{g}} r_{1}^{-2}\left(\mathbf{r}^{\prime} \overline{\mathbf{J}}_{11} \overline{\mathbf{D}} \overline{\mathbf{F}} \overline{\mathbf{A}}_{L} \overline{\mathbf{G}}^{\prime} \overline{\mathbf{C}}^{\prime} \mathbf{r}\right)\right| \phi_{L}\right\rangle-2 m_{K}\left\langle\phi_{K}\left|\frac{1}{r_{g}} r_{1}^{-2}\left(\mathbf{r}^{\prime} \overline{\mathbf{J}}_{11} \overline{\mathbf{D}} \overline{\mathbf{F}} \overline{\mathbf{A}}_{L} \overline{\mathbf{G}}^{\prime} \overline{\mathbf{C}}^{\prime} \mathbf{r}\right)\right| \phi_{L}\right\rangle \\
& -8\left\langle\phi_{K}\left|\frac{1}{r_{g}}\left(\mathbf{r}^{\prime} \overline{\mathbf{C}} \overline{\mathbf{G}} \overline{\mathbf{A}}_{L} \mathbf{r}\right)\left(\mathbf{r}^{\prime} \overline{\mathbf{A}}_{K} \overline{\mathbf{D}} \overline{\mathbf{F}} \overline{\mathbf{A}}_{L} \mathbf{r}\right)\right| \phi_{L}\right\rangle+4\left\langle\phi_{K}\left|\frac{1}{r_{g}}\left(\mathbf{r}^{\prime} \overline{\mathbf{A}}_{K} \overline{\mathbf{D}} \overline{\mathbf{F}} \overline{\mathbf{A}}_{L} \overline{\mathbf{G}}^{\prime} \overline{\mathbf{C}}^{\prime} \mathbf{r}\right)\right| \phi_{L}\right\rangle .
\end{aligned}
$$

(2) $\operatorname{Term} 2 \phi_{K}\left\{\left(\nabla^{\prime} \overline{\mathbf{D}}\right)^{\beta}\left(\mathbf{r}^{\prime} \overline{\mathbf{C}}\right)^{\alpha}\right\}(\overline{\mathbf{F}} \nabla)_{\beta}(\overline{\mathbf{G}} \nabla)_{\alpha} \phi_{L}$

$$
\begin{aligned}
\left(\nabla^{\prime} \overline{\mathbf{D}}\right)^{\beta}\left(\mathbf{r}^{\prime} \overline{\mathbf{C}}\right)^{\alpha}=\overline{\mathbf{D}}_{\gamma}^{\beta} \cdot \nabla^{\gamma} \mathbf{r}^{\rho} \overline{\mathbf{C}}_{\rho}^{\alpha} & =\overline{\mathbf{D}}_{\gamma}^{\beta} \delta^{\gamma \rho} \overline{\mathbf{C}}_{\rho}^{\alpha} \\
& =\overline{\mathbf{D}}_{\gamma}^{\beta} \overline{\mathbf{C}}^{\gamma \alpha}\left\{\left(\nabla^{\prime} \overline{\mathbf{D}}\right)^{\beta}\left(\mathbf{r}^{\prime} \overline{\mathbf{C}}\right)^{\alpha}\right\}(\overline{\mathbf{F}} \nabla)_{\beta}(\overline{\mathbf{G}} \nabla)_{\alpha} \phi_{L} \\
& =\overline{\mathbf{D}}_{\gamma}^{\beta}(\overline{\mathbf{F}} \nabla)_{\beta}(\overline{\mathbf{C}} \overline{\mathbf{G}} \nabla)^{\gamma} \phi_{L}=\left(\nabla^{\prime} \overline{\mathbf{G}}^{\prime} \overline{\mathbf{C}} \overline{\mathbf{D}}^{\prime} \overline{\mathbf{D}} \nabla\right) \phi_{L} .
\end{aligned}
$$

In this way we obtain an expression analogical to Appendix A 2. Now, since $\overline{\mathbf{M}}=\overline{\mathbf{G}}^{\prime} \overline{\mathbf{C}}^{\prime} \overline{\mathbf{D}} \overline{\mathbf{F}}$ we get

$$
\begin{aligned}
& \nabla^{\prime} \overline{\mathbf{M}} \nabla \phi_{L}=r_{1}^{-4} m_{L}\left(m_{L}-2\right)\left(\mathbf{r}^{\prime} \overline{\mathbf{J}}_{11} \overline{\mathbf{M}} \overline{\mathbf{J}}_{11} \mathbf{r}\right) \phi_{L}-2 r_{1}^{-2} m_{L}\left[\left(\mathbf{r}^{\prime} \overline{\mathbf{A}}_{L} \overline{\mathbf{M}}_{\mathbf{J}} \overline{\mathbf{J}}_{11} \mathbf{r}\right)\right. \\
& \left.+\left(\mathbf{r}^{\prime} \overline{\mathbf{J}}_{11} \overline{\mathbf{M}} \overline{\mathbf{A}}_{L} \mathbf{r}\right)\right] \phi_{L}+r_{1}^{-2} 3 m_{L} \operatorname{tr}\left[\mathbf{M} \mathbf{J}_{11}\right] \phi_{L}+4\left(\mathbf{r}^{\prime} \overline{\mathbf{A}}_{L} \overline{\mathbf{M}} \overline{\mathbf{A}}_{L} \mathbf{r}\right) \phi_{L}-6 \operatorname{tr}\left[\mathbf{A}_{L} \mathbf{M}\right] \phi_{L} .
\end{aligned}
$$

Now, we can evaluate the expectation value of term 2 as

$$
\begin{aligned}
\left\langle\phi_{K}\right| & \frac{1}{r_{g}}\left\{\left(\nabla^{\prime} \overline{\mathbf{D}}\right)^{\beta}\left(\mathbf{r}^{\prime} \overline{\mathbf{C}}\right)^{\alpha}\right\}(\overline{\mathbf{F}} \nabla)_{\beta}(\overline{\mathbf{G}} \nabla)_{\alpha}\left|\phi_{L}\right\rangle \\
= & m_{L}\left(m_{L}-2\right)\left\langle\phi_{K}\left|\frac{1}{r_{g}} r_{1}^{-4}\left(\mathbf{r}^{\prime} \overline{\mathbf{J}}_{11} \overline{\mathbf{G}}^{\prime} \overline{\mathbf{C}}^{\prime} \overline{\mathbf{D}} \overline{\mathbf{F}} \overline{\mathbf{J}}_{11} \mathbf{r}\right)\right| \phi_{L}\right\rangle-2 m_{L}\left\langle\phi_{K}\left|\frac{1}{r_{\beta}} r_{1}^{-2}\left[\left(\mathbf{r}^{\prime} \overline{\mathbf{A}}_{L} \overline{\mathbf{G}}^{\prime} \overline{\mathbf{C}}^{\prime} \overline{\mathbf{D}} \overline{\mathbf{F}} \overline{\mathbf{J}}_{11} \mathbf{r}\right)+\left(\mathbf{r}^{\prime} \overline{\mathbf{J}}_{11} \overline{\mathbf{G}}^{\prime} \overline{\mathbf{C}}^{\prime} \overline{\mathbf{D}} \overline{\mathbf{F}} \overline{\mathbf{A}}_{L} \mathbf{r}\right)\right]\right| \phi_{L}\right\rangle \\
& +3 m_{L} \operatorname{tr}\left[\mathbf{G}^{\prime} \mathbf{C}^{\prime} \mathbf{D F} \mathbf{J}_{11}\right]\left\langle\phi_{K}\left|\frac{1}{r_{g}} r_{1}^{-2}\right| \phi_{L}\right\rangle+4\left\langle\phi_{K}\left|\frac{1}{r_{g}}\left(\mathbf{r}^{\prime} \overline{\mathbf{A}}_{L} \overline{\mathbf{G}}^{\prime} \overline{\mathbf{C}}^{\prime} \overline{\mathbf{D}} \overline{\mathbf{F}} \overline{\mathbf{A}}_{L} \mathbf{r}\right)\right| \phi_{L}\right\rangle 6 \operatorname{tr}\left[\mathbf{A}_{L} \mathbf{G}^{\prime} \mathbf{C}^{\prime} \mathbf{D F}\right]\left\langle\phi_{K}\left|\frac{1}{r_{g}}\right| \phi_{L}\right\rangle
\end{aligned}
$$


(3) $\operatorname{Term} 3 \phi_{K}\left(\mathbf{r}^{\prime} \overline{\mathbf{C}}\right)^{\alpha}\left\{\left(\nabla^{\prime} \overline{\mathbf{D}}\right)^{\beta}(\overline{\mathbf{F}} \nabla)_{\beta}(\overline{\mathbf{G}} \nabla)_{\alpha} \phi_{L}\right\}$

First we make a few simple transformations,

$\left(\mathbf{r}^{\prime} \overline{\mathbf{C}}\right)^{\alpha}\left\{\left(\nabla^{\prime} \overline{\mathbf{D}}\right)^{\beta}(\overline{\mathbf{F}} \nabla)_{\beta}(\overline{\mathbf{G}} \nabla)_{\alpha} \phi_{L}\right\}=\left(\mathbf{r}^{\prime} \overline{\mathbf{C}} \overline{\mathbf{G}}\right)^{\alpha}(\overline{\mathbf{D}} \overline{\mathbf{F}})_{\rho}^{\beta} \nabla^{\rho} \nabla_{\alpha} \nabla_{\beta} \phi_{L}$,

where the following simple relation was used:

$\left(\mathbf{r}^{\prime} \overline{\mathbf{C}}\right)^{\alpha}(\overline{\mathbf{G}} \nabla)_{\alpha}\left(\nabla^{\prime} \overline{\mathbf{D}}\right)^{\beta}(\overline{\mathbf{F}} \nabla)_{\beta} \phi_{L}=\left(\mathbf{r}^{\prime} \overline{\mathbf{C}} \overline{\mathbf{G}} \nabla\right)\left(\nabla^{\prime} \overline{\mathbf{D}} \overline{\mathbf{F}} \nabla\right) \phi_{L}=\left(\mathbf{r}^{\prime} \overline{\mathbf{C}} \overline{\mathbf{G}}\right)^{\alpha}(\overline{\mathbf{D}} \overline{\mathbf{F}})_{\rho}^{\beta} \nabla^{\rho} \nabla_{\alpha} \nabla_{\beta} \phi_{L}$

The whole task of evaluating the integral now reduces to determining $\nabla^{\rho} \nabla_{\alpha} \nabla_{\beta} \phi_{L}$. This can be easily calculated using (A4) and (A5). Performing some tedious elementary transformations we get

$$
\begin{aligned}
& \left(\mathbf{r}^{\prime} \overline{\mathbf{C}} \overline{\mathbf{G}}\right)^{\alpha}(\overline{\mathbf{D}} \overline{\mathbf{F}})_{\rho}^{\beta} \nabla^{\rho} \nabla_{\alpha} \nabla_{\beta} \phi_{L} \\
& =r_{1}^{-6} m_{L}\left(m_{L}-2\right)\left(m_{L}-4\right)\left(\mathbf{r}^{\prime} \overline{\mathbf{C}} \overline{\mathbf{G}} \overline{\mathbf{J}}_{11} \mathbf{r}\right)\left(\mathbf{r}^{\prime} \mathbf{J}_{11} \overline{\mathbf{D}} \overline{\mathbf{F}} \overline{\mathbf{J}}_{11} \mathbf{r}\right) \phi_{L}+r_{1}^{-4} m_{L}\left(m_{L}-2\right)\left[\left(\mathbf{r}^{\prime} \overline{\mathbf{C}} \overline{\mathbf{G}}_{\mathbf{J}_{11}} \overline{\mathbf{D}} \overline{\mathbf{F}} \overline{\mathbf{J}}_{11} \mathbf{r}\right)+3 \operatorname{tr}\left[\mathbf{D F} \mathbf{J}_{11}\right]\left(\mathbf{r}^{\prime} \overline{\mathbf{C}} \overline{\mathbf{G}} \overline{\mathbf{J}}_{11}\right)\right. \\
& \left.+\left(\mathbf{r}^{\prime} \overline{\mathbf{C}} \overline{\mathbf{G}}_{\mathbf{J}_{11}} \mathbf{F}^{\prime} \overline{\mathbf{D}}^{\prime} \overline{\mathbf{J}}_{11} \mathbf{r}\right)\right] \phi_{L}+r_{1}^{-4}(-2) m_{L}\left(m_{L}-2\right)\left[\left(\mathbf{r}^{\prime} \overline{\mathbf{C}} \overline{\mathbf{G}} \overline{\mathbf{J}}_{11} \mathbf{r}\right)\left(\mathbf{r}^{\prime} \overline{\mathbf{A}}_{L} \overline{\mathbf{D}} \overline{\mathbf{F}} \overline{\mathbf{J}}_{11} \mathbf{r}\right)+\left(\mathbf{r}, \overline{\mathbf{C}} \overline{\mathbf{G}} \overline{\mathbf{J}}_{11} \mathbf{r}\right)\left(\mathbf{r}^{\prime} \overline{\mathbf{J}}_{11} \overline{\mathbf{D}} \overline{\mathbf{F}} \overline{\mathbf{A}}_{L} \mathbf{r}\right)\right. \\
& \left.+\left(\mathbf{r}^{\prime} \overline{\mathbf{C}} \overline{\mathbf{G}} \overline{\mathbf{A}}_{L} \mathbf{r}\right)\left(\mathbf{r}^{\prime} \overline{\mathbf{J}}_{11} \overline{\mathbf{D}} \overline{\mathbf{F}} \overline{\mathbf{J}}_{11} \mathbf{r}\right)\right] \phi_{L}+r_{1}^{-2} 4 m_{L}\left[\left(\mathbf{r}^{\prime} \overline{\mathbf{C}} \overline{\mathbf{G}} \overline{\mathbf{J}}_{11} \mathbf{r}\right)\left(\mathbf{r}^{\prime} \overline{\mathbf{A}}_{L} \overline{\mathbf{D}} \overline{\mathbf{F}} \overline{\mathbf{A}}_{L} \mathbf{r}\right)+\left(\mathbf{r}^{\prime} \overline{\mathbf{C}} \overline{\mathbf{G}}_{\overline{\mathbf{A}}} \mathbf{r}\right)\left(\mathbf{r}^{\prime} \overline{\mathbf{A}}_{L} \overline{\mathbf{D}}_{\overline{\mathbf{F}}} \overline{\mathbf{J}}_{11} \mathbf{r}\right)\right.
\end{aligned}
$$

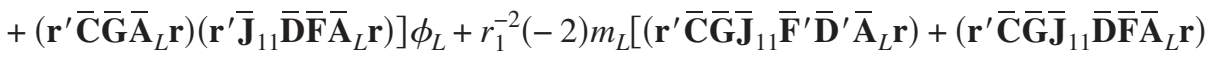

$$
\begin{aligned}
& \left.+3 \operatorname{tr}\left[\mathbf{D F} \mathbf{J}_{11}\right]\left(\mathbf{r}^{\prime} \overline{\mathbf{C}} \overline{\mathbf{G}}_{L_{L}} \mathbf{r}\right)\right] \phi_{L}+r_{1}^{-2}(-2) m_{L}\left[\left(\mathbf{r}^{\prime} \overline{\mathbf{C}} \overline{\mathbf{G}}_{L} \overline{\mathbf{F}}^{\prime} \overline{\mathbf{D}}^{\prime} \overline{\mathbf{J}}_{11} \mathbf{r}\right)+3 \operatorname{tr}\left[\dot{\mathbf{D F}} \mathbf{A}_{L}\right]\left(\mathbf{r}^{\prime} \overline{\mathbf{C}} \overline{\mathbf{G}}_{11} \mathbf{r}\right)\right. \\
& \left.+\left(\mathbf{r}^{\prime} \overline{\mathbf{C}} \overline{\mathbf{G}}_{\overline{\mathbf{A}}} \overline{\mathbf{D}} \overline{\mathbf{F}} \overline{\mathbf{J}}_{11} \mathbf{r}\right)\right] \phi_{L}+4\left[\left(\mathbf{r}^{\prime} \overline{\mathbf{C}} \overline{\mathbf{C}}_{L} \overline{\mathbf{D}} \overline{\mathbf{F}} \overline{\boldsymbol{\Lambda}}_{L} \mathbf{r}\right)+3 \operatorname{tr}\left[\mathbf{D F} \mathbf{A}_{L}\right]\left(\mathbf{r}^{\prime} \overline{\mathbf{C}} \overline{\mathbf{G}}_{\mathbf{A}_{L}} \mathbf{r}\right)+\left(\mathbf{r}^{\prime} \overline{\mathbf{C}} \overline{\mathbf{G}}_{L} \overline{\mathbf{A}}_{L} \overline{\mathbf{F}}^{\prime} \overline{\mathbf{D}}^{\prime} \overline{\mathbf{A}}_{L} \mathbf{r}\right)\right] \phi_{L} \\
& -8\left(\mathbf{r}^{\prime} \overline{\mathbf{C}} \overline{\mathbf{G}}_{\overline{\mathbf{A}}_{L}} \mathbf{r}\right)\left(\mathbf{r}^{\prime} \overline{\mathbf{A}}_{L} \overline{\mathbf{D}} \overline{\mathbf{F}} \overline{\mathbf{A}}_{L} \mathbf{r}\right) \phi_{L} \text {. }
\end{aligned}
$$

The above three relations allow us to evaluate term 3 as

$$
\begin{aligned}
& \left\langle\phi_{K}\left|\left(\mathbf{r}^{\prime} \overline{\mathbf{C}}\right)^{\alpha}\left(\nabla^{\prime} \overline{\mathbf{D}}\right)^{\beta}(\overline{\mathbf{F}} \nabla)_{\beta}(\overline{\mathbf{G}} \nabla)_{\alpha}\right| \phi_{L}\right\rangle \\
& =m_{L}\left(m_{L}-2\right)\left(m_{L}-4\right)\left\langle\phi_{K}\left|\frac{1}{r_{g}} r_{1}^{-6}\left(\mathbf{r}^{\prime} \overline{\mathbf{C}} \overline{\mathbf{G}}_{\mathbf{J}} \overline{\mathbf{J}}_{11} \mathbf{r}\right)\left(\mathbf{r}^{\prime} \overline{\mathbf{J}}_{11} \overline{\mathbf{D}} \overline{\mathbf{F}} \overline{\mathbf{J}}_{11} \mathbf{r}\right)\right| \phi_{L}\right\rangle+m_{L}\left(m_{L}-2\right)\left\langle\phi_{K}\right| \frac{1}{r_{g}} r_{1}^{-4}\left[\left(\mathbf{r}^{\prime} \overline{\mathbf{C}} \overline{\mathbf{G}} \overline{\mathbf{J}}_{11} \overline{\mathbf{D}} \overline{\mathbf{F}} \overline{\mathbf{J}}_{11} \mathbf{r}\right)\right. \\
& \left.+\left(\mathbf{r}^{\prime} \overline{\mathbf{C}} \overline{\mathbf{G}}_{\mathbf{J}_{11}} \overline{\mathbf{F}}^{\prime} \overline{\mathbf{D}}^{\prime} \overline{\mathbf{J}}_{11} \mathbf{r}\right)\right]\left|\phi_{L}\right\rangle+3 \operatorname{tr}\left[\mathbf{D F} \mathbf{J}_{11}\right] m_{L}\left(m_{L}-2\right)\left\langle\phi_{K}\left|\frac{1}{r_{g}} r_{1}^{-4}\left(\mathbf{r}^{\prime} \overline{\mathbf{C}} \overline{\mathbf{G}} \overline{\mathbf{J}}_{11}\right)\right| \phi_{L}\right\rangle \\
& -2 m_{L}\left(m_{L}-2\right)\left\langle\phi_{K}\left|\frac{1}{r_{g}} r_{1}^{-4}\left(\mathbf{r}^{\prime} \overline{\mathbf{C}} \overline{\mathbf{G}} \overline{\mathbf{J}}_{11} \mathbf{r}\right)\left[\left(\mathbf{r}^{\prime} \overline{\mathbf{A}}_{L} \overline{\mathbf{D}} \overline{\mathbf{F}} \overline{\mathbf{J}}_{11} \mathbf{r}\right)+\left(\mathbf{r}^{\prime} \overline{\mathbf{J}}_{11} \overline{\mathbf{D}} \overline{\mathbf{F}} \overline{\mathbf{A}}_{L} \mathbf{r}\right)\right]\right| \phi_{L}\right\rangle \\
& -2 m_{L}\left(m_{L}-2\right)\left\langle\phi_{K}\left|\frac{1}{r_{g}} r_{1}^{-4}\left(\mathbf{r}^{\prime} \overline{\mathbf{C}} \overline{\mathbf{G}} \overline{\mathbf{A}}_{L} \mathbf{r}\right)\left(\mathbf{r}^{\prime} \mathbf{J}_{11} \overline{\mathbf{D}} \overline{\mathbf{F}} \overline{\mathbf{J}}_{11} \mathbf{r}\right)\right| \phi_{L}\right\rangle+4 m_{L}\left\langle\phi_{K}\right| \frac{1}{r_{g}} r_{1}^{-2}\left(\mathbf{r}^{\prime} \overline{\mathbf{C}} \overline{\mathbf{G}}_{\mathbf{A}_{L}} \mathbf{r}\right)\left[\left(\mathbf{r}^{\prime} \overline{\mathbf{A}}_{L} \overline{\mathbf{D}} \overline{\mathbf{F}} \overline{\mathbf{J}}_{11} \mathbf{r}\right)\right. \\
& \left.+\left(\mathbf{r}^{\prime} \overline{\mathbf{J}}_{11} \overline{\mathbf{D}} \overline{\mathbf{F}} \overline{\mathbf{A}}_{L} \mathbf{r}\right)\right]\left|\phi_{L}\right\rangle+4 m_{L}\left\langle\phi_{K}\left|\frac{1}{r_{g}} r_{1}^{-2}\left(\mathbf{r}^{\prime} \overline{\mathbf{C}} \overline{\mathbf{G}} \overline{\mathbf{J}}_{11} \mathbf{r}\right)\left(\mathbf{r}^{\prime} \overline{\mathbf{A}}_{L} \overline{\mathbf{D}} \overline{\mathbf{F}}_{\overline{\mathbf{A}}} \overline{\mathbf{A}}_{L} \mathbf{r}\right)\right| \phi_{L}\right\rangle-2 m_{L}\left\langle\phi_{K}\right| \frac{1}{r_{g}} r_{1}^{-2}\left[\left(\mathbf{r}^{\prime} \overline{\mathbf{C}} \overline{\mathbf{G}} \overline{\mathbf{J}}_{11} \overline{\mathbf{F}}^{\prime} \overline{\mathbf{D}}^{\prime} \overline{\mathbf{A}}_{L} \mathbf{r}\right)\right. \\
& \left.+\left(\mathbf{r}^{\prime} \overline{\mathbf{C}} \overline{\mathbf{G}} \overline{\mathbf{J}}_{11} \overline{\mathbf{D}} \overline{\mathbf{F}} \overline{\mathbf{A}}_{L} \mathbf{r}\right)\right]\left|\phi_{L}\right\rangle-6 m_{L} \operatorname{tr}\left[\mathbf{D F} \mathbf{J}_{11}\right]\left\langle\phi_{K}\left|\frac{1}{r_{g}} r_{1}^{-2}\left(\mathbf{r}^{\prime} \overline{\mathbf{C}} \overline{\mathbf{G}}_{L} \overline{\mathbf{A}}_{L} \mathbf{r}\right)\right| \phi_{L}\right\rangle-2 m_{L}\left\langle\phi_{K}\right| \frac{1}{r_{g}} r_{1}^{-2}\left[\left(\mathbf{r}^{\prime} \overline{\mathbf{C}} \overline{\mathbf{G}} \overline{\mathbf{A}}_{L} \overline{\mathbf{F}}^{\prime} \overline{\mathbf{D}}^{\prime} \overline{\mathbf{J}}_{11} \mathbf{r}\right)\right. \\
& \left.+\left(\mathbf{r}^{\prime} \overline{\mathbf{C}} \overline{\mathbf{G}}_{L} \overline{\mathbf{A}}_{L} \overline{\mathbf{D}} \overline{\mathbf{F}} \overline{\mathbf{J}}_{11} \mathbf{r}\right)\right] \phi_{L}-6 m_{L} \operatorname{tr}\left[\mathbf{D F} \mathbf{A}_{L}\right]\left\langle\phi_{K}\left|\frac{1}{r_{g}} r_{1}^{-2}\left(\mathbf{r}^{\prime} \overline{\mathbf{C}} \overline{\mathbf{G}} \overline{\mathbf{J}}_{11} \mathbf{r}\right)\right| \phi_{L}\right\rangle+4\left\langle\phi_{K}\right| \frac{1}{r_{g}}\left[\left(\mathbf{r}^{\prime} \overline{\mathbf{C}} \overline{\mathbf{G}} \overline{\mathbf{A}}_{L} \overline{\mathbf{D}} \overline{\mathbf{F}} \overline{\mathbf{A}}_{L} \mathbf{r}\right)\right. \\
& \left.+\left(\mathbf{r}^{\prime} \overline{\mathbf{C}} \overline{\mathbf{G}} \overline{\mathbf{A}}_{L} \overline{\mathbf{F}}^{\prime} \overline{\mathbf{D}}^{\prime} \overline{\mathbf{A}}_{L} \mathbf{r}\right)\right]\left|\phi_{L}\right\rangle+12 \operatorname{tr}\left[\mathbf{D F} \mathbf{A}_{L}\right]\left\langle\phi_{K}\left|\frac{1}{r_{g}}\left(\mathbf{r}^{\prime} \overline{\mathbf{C}} \overline{\mathbf{G}} \overline{\mathbf{A}}_{L} \mathbf{r}\right)\right| \phi_{L}\right\rangle-8\left\langle\phi_{K}\left|\frac{1}{r_{g}}\left(\mathbf{r}^{\prime} \overline{\mathbf{C}} \overline{\mathbf{G}} \overline{\mathbf{A}}_{L} \mathbf{r}\right)\left(\mathbf{r}^{\prime} \overline{\mathbf{A}}_{L} \overline{\mathbf{D}} \overline{\mathbf{F}} \overline{\mathbf{A}}_{L} \mathbf{r}\right)\right| \phi_{L}\right\rangle .
\end{aligned}
$$


The integrals that appear in the expression for term 1 , term 2 , and term 3 were either derived in our previous work ${ }^{16}$ or are derived in Appendix B in this work.

By adding the quantities term 1, term 2, and term 3 we get the following expression for the integral:

$$
\begin{aligned}
&\left\langle\phi_{K}\right| \frac{1}{r_{g}} \nabla^{\prime} \overline{\mathbf{B}} \nabla\left|\phi_{L}\right\rangle-\left\langle\phi_{K}\right| \\
& \quad \times\left(\mathbf{r}^{\prime} \overline{\mathbf{C}}\right)^{\alpha}\left(\nabla^{\prime} \overline{\mathbf{D}} \frac{1}{r_{g}}\right)^{\beta}(\overline{\mathbf{F}} \nabla)_{\beta}(\overline{\mathbf{G}} \nabla)_{\alpha}\left|\phi_{L}\right\rangle .
\end{aligned}
$$

Next, substituting for matrices $\overline{\mathbf{B}}, \overline{\mathbf{C}}, \overline{\mathbf{D}}, \overline{\mathbf{F}}$, and $\overline{\mathbf{G}}$ the quantities from Eq. (A10), we get the final expressions for the terms involved in the expectation value of the orbit-orbit interaction operator. To complete the task we notice the following relations concerning quantities in Eq. (A10): $\overline{\mathbf{C G}}$ $=\overline{\mathbf{C}}$ and $\overline{\mathbf{D F}}=\overline{\mathbf{D}}$. These relations allow simplification of the final expression for the matrix element of the orbit-orbit interaction operator.

\section{APPENDIX B: INTEGRAL $\left\langle\phi_{K}\left|r_{1}^{-q} / r_{g}\left(r^{\prime} \overline{\mathrm{B}} r\right)\left(r^{\prime} \overline{\mathrm{C}} r\right)\right| \phi_{L}\right\rangle$}

The calculation of the matrix elements of the $\hat{H}_{O O}$ operator requires evaluation of the following types of integrals:

- $\left\langle\phi_{K}\left|r_{1}^{-q} / r_{g}\left(\mathbf{r}^{\prime} \overline{\mathbf{B}} \mathbf{r}\right)\right| \phi_{L}\right\rangle$,

- $\left\langle\phi_{K}\left|r_{1}^{-q} / r_{g}\left(\mathbf{r}^{\prime} \overline{\mathbf{B}} \mathbf{r}\right)\left(\mathbf{r}^{\prime} \overline{\mathbf{C}} \mathbf{r}\right)\right| \phi_{L}\right\rangle$.

The first type of the integral already appeared in the calculation of the expectation value of the $H_{D}$ operator (A6). The second one is a new type of integral whose derivation is presented next.

This integral is evaluated using the following relation taken from the work of Kinghorn: ${ }^{36}$

$$
\left\langle\phi_{K}\left|r_{1}^{-q} \frac{1}{r_{g}}\left(\mathbf{r}^{\prime} \overline{\mathbf{B}} \mathbf{r}\right)\left(\mathbf{r}^{\prime} \overline{\mathbf{C}} \mathbf{r}\right)\right| \phi_{L}\right\rangle=\left\langle\phi_{K}\left|r_{1}^{-q} \frac{1}{r_{g}} \mathbf{r}^{\alpha} \mathbf{r}_{\beta} \mathbf{r}^{\rho} \mathbf{r}_{\gamma}\right| \phi_{L}\right\rangle \overline{\mathbf{B}}_{\alpha}^{\beta} \overline{\mathbf{C}}_{\rho}^{\gamma}=\overline{\mathbf{B}}_{\alpha}^{\beta} \overline{\mathbf{C}}_{\rho}^{\gamma}\left\{\partial_{\alpha \beta} \partial_{\rho \gamma}\left\langle\phi_{K}\left|r_{1}^{-q} \frac{1}{r_{g}}\right| \phi_{L}\right\rangle\right\},
$$

where the notation $\partial_{\alpha \beta}$ denotes

$$
\frac{\partial}{\partial\left(\overline{\mathbf{A}}_{k l}\right)_{\alpha \beta}} \equiv \partial_{\alpha \beta}
$$

In the derivation of the integral we make use of the following previously derived relations:

(I) $\partial_{\rho \gamma}\left(\overline{\mathbf{A}}^{-1}\right)_{\alpha \beta}=-\overline{\mathbf{A}}_{\alpha \rho}^{-1} \overline{\mathbf{A}}_{\beta \gamma}^{-1}$,

(II) $\partial_{\rho \gamma}\left(\overline{\mathbf{C}} \overline{\mathbf{A}}^{-1}\right)_{\alpha \beta}=\overline{\mathbf{C}}_{\alpha}^{\xi} \partial_{\rho \gamma} \overline{\mathbf{A}}_{\xi \beta}^{-1}=-\left(\overline{\mathbf{C}} \overline{\mathbf{A}}^{-1}\right)_{\alpha \rho} \overline{\mathbf{A}}_{\beta \gamma}^{-1}$,

(III) $\quad \partial_{\rho \gamma}\left(\overline{\mathbf{A}}^{-1} \overline{\mathbf{B}} \overline{\mathbf{A}}^{-1}\right)_{\alpha \beta}=-\left[\left(\overline{\mathbf{A}}_{k l}^{-1}\right)_{\sigma \rho}\left(\overline{\mathbf{A}}_{k l}^{-1} \overline{\mathbf{B}} \overline{\mathbf{A}}_{k l}^{-1}\right)_{\gamma \beta}+\left(\overline{\mathbf{A}}_{k l}^{-1} \overline{\mathbf{B}}_{\mathbf{\mathbf { A }}}^{-1}\right)_{\alpha \rho}\left(\overline{\mathbf{A}}_{k l}^{-1}\right)_{\gamma \beta}\right]$,

(IV) $\partial_{\rho \gamma}\left(\overline{\mathbf{A}}^{-1} \overline{\mathbf{B}} \overline{\mathbf{A}}^{-1} \overline{\mathbf{C}} \overline{\mathbf{A}}^{-1}\right)_{\alpha \beta}=\partial_{\rho \gamma}\left[\left(\overline{\mathbf{A}}^{-1} \overline{\mathbf{B}} \overline{\mathbf{A}}^{-1}\right)_{\alpha}^{\xi}\left(\overline{\mathbf{C}} \overline{\mathbf{A}}^{-1}\right)_{\xi \beta}\right]=-\left[\overline{\mathbf{A}}_{\alpha \rho}^{-1}\left(\overline{\mathbf{A}}^{-1} \overline{\mathbf{B}} \overline{\mathbf{A}}^{-1} \overline{\mathbf{C}}^{-1}\right)_{\gamma \beta}+\left(\overline{\mathbf{A}}^{-1} \overline{\mathbf{B}} \overline{\mathbf{A}}^{-1}\right)_{\alpha \rho}\left(\overline{\mathbf{A}}^{-1} \overline{\mathbf{C}} \overline{\mathbf{A}}^{-1}\right)_{\gamma \beta}\right.$

$$
\left.+\left(\overline{\mathbf{A}}^{-1} \overline{\mathbf{B}} \overline{\mathbf{A}}^{-1} \overline{\mathbf{C}}^{-1}\right)_{\alpha \rho} \overline{\mathbf{A}}_{\gamma \beta}^{-1}\right]
$$

(V) $\partial_{\alpha \beta} \operatorname{tr}\left[\overline{\mathbf{B}} \overline{\mathbf{A}}_{k l}^{-1}\right]=-\left(\overline{\mathbf{A}}_{k l}^{-1} \overline{\mathbf{B}} \overline{\mathbf{A}}_{k l}^{-1}\right)_{\alpha \beta}$,

$$
\partial_{\rho \gamma} \operatorname{tr}\left[\overline{\mathbf{B}} \overline{\mathbf{A}}^{-1} \overline{\mathbf{C}} \overline{\mathbf{A}}^{-1}\right]=\overline{\mathbf{B}}_{\xi}^{\alpha} \partial_{\rho \gamma}\left(\overline{\mathbf{A}}^{-1} \overline{\mathbf{C}} \overline{\mathbf{A}}^{-1}\right)_{\alpha}^{\xi}=-\left[\left(\overline{\mathbf{A}}^{-1} \overline{\mathbf{C}} \overline{\mathbf{A}}^{-1} \overline{\mathbf{B}} \overline{\mathbf{A}}^{-1}\right)_{\rho \gamma}+\left(\overline{\mathbf{A}}^{-1} \overline{\mathbf{B}} \overline{\mathbf{A}}^{-1} \overline{\mathbf{C}} \overline{\mathbf{A}}^{-1}\right)_{\rho \gamma}\right]
$$

$$
\partial_{\alpha \beta}\left\langle\phi_{k}\left|r_{1}^{-q}\right| \phi_{l}\right\rangle=-\left\langle\phi_{k}\left|r_{1}^{-q}\right| \phi_{l}\right\rangle\left[m_{m k}-q / 2 \operatorname{tr}^{-1}\left[\overline{\mathbf{J}}_{11} \overline{\mathbf{A}}_{k l}^{-1}\right]\left(\overline{\mathbf{A}}_{k l}^{-1} \overline{\mathbf{J}}_{11} \overline{\mathbf{A}}_{k l}^{-1}\right)_{\alpha \beta}+\frac{1}{2}\left(\overline{\mathbf{A}}_{k l}^{-1}\right)_{\alpha \beta}\right]
$$

$$
\begin{aligned}
\partial_{\alpha \beta}\left\langle\phi_{k}\left|r_{1}^{-q} 1 / r_{g}\right| \phi_{l}\right\rangle= & -\frac{1}{2}\left\langle\phi_{k}\left|r_{1}^{-q} 1 / r_{g}\right| \phi_{l}\right\rangle\left[2 p / \bar{a}\left(\overline{\mathbf{A}}_{k l}^{-1} \overline{\mathbf{J}}_{11} \overline{\mathbf{A}}_{k l}^{-1}\right)_{\alpha \beta}+\left(\overline{\mathbf{A}}_{k l}^{-1}\right)_{\alpha \beta}-1 / \bar{b}\left(\overline{\mathbf{A}}_{k l}^{-1} \overline{\mathbf{J}}_{g} \overline{\mathbf{A}}_{k l}^{-1}\right)_{\alpha \beta}\right] \\
& +\left\langle\phi_{k}\left|r_{1}^{-q}\right| \phi_{l}\right\rangle 3 \sqrt{3} \gamma_{2}(p) / \bar{a}^{2} \bar{b}^{2} \sqrt{\bar{b}} \sum_{s=1}^{p} \gamma_{3}(s) s(1-3 \bar{c} / \bar{a} \bar{b})^{s-1}\left[\bar{a} \bar{b}\left(\overline{\mathbf{A}}_{k l}^{-1} \overline{\mathbf{J}}_{11} \overline{\mathbf{A}}_{k l}^{-1} \overline{\mathbf{J}}_{g} \overline{\mathbf{A}}_{k l}^{-1}\right)_{\alpha \beta}\right. \\
& \left.+\bar{a} \bar{b}\left(\overline{\mathbf{A}}_{k l}^{-1} \overline{\mathbf{J}}_{g} \overline{\mathbf{A}}_{k l}^{-1} \overline{\mathbf{J}}_{11} \overline{\mathbf{A}}_{k l}^{-1}\right)_{\alpha \beta}-\overline{a c}\left(\overline{\mathbf{A}}_{k l}^{-1} \overline{\mathbf{J}}_{g} \overline{\mathbf{A}}_{k l}^{-1}\right)_{\alpha \beta}-\bar{b} \bar{c}\left(\overline{\mathbf{A}}_{k l}^{-1} \overline{\mathbf{J}}_{11} \overline{\mathbf{A}}_{k l}^{-1}\right)_{\alpha \beta}\right]
\end{aligned}
$$

(for details see Refs. 16 and 38).

First we need to calculate the second derivative of the integral $\left\langle\phi_{k}\left|r_{1}^{-q} 1 / r_{g}\right| \phi_{l}\right\rangle$. According to (VIII) the expression for the derivative can be written as 


$$
\begin{aligned}
& \overline{\mathbf{B}}_{\alpha}^{\beta} \overline{\mathbf{C}}_{\rho}^{\gamma} \partial_{\rho \gamma}\left\{\partial_{\alpha \beta}\left\langle\phi_{k}\left|r_{1}^{-q} \frac{1}{r_{g}}\right| \phi_{l}\right\rangle\right\} \\
& =-\frac{1}{2} \overline{\mathbf{B}}_{\alpha}^{\beta} \overline{\mathbf{C}}_{\rho}^{\gamma} \partial_{\rho \gamma}\left\{\left\langle\phi_{k}\left|r_{1}^{-q} \frac{1}{r_{g}}\right| \phi_{l}\right\rangle\left[\frac{m_{k l-q}}{\bar{a}}\left(\overline{\mathbf{A}}_{k l}^{-1} \overline{\mathbf{J}}_{11} \overline{\mathbf{A}}_{k l}^{-1}\right)_{\alpha \beta}+\left(\overline{\mathbf{A}}_{k l}^{-1}\right)_{\alpha \beta}-\frac{1}{\bar{b}}\left(\overline{\mathbf{A}}_{k l}^{-1} \overline{\mathbf{J}}_{g} \overline{\mathbf{A}}_{k l}^{-1}\right)_{\alpha \beta}\right]\right\} \\
& +\overline{\mathbf{B}}_{\alpha}^{\beta} \overline{\mathbf{C}}_{\rho}^{\gamma} \partial_{\rho \gamma}\left\{\langle \phi _ { k } | r _ { 1 } ^ { - q } | \phi _ { l } \rangle \frac { 3 \sqrt { 3 } \gamma _ { 2 } ( p ) } { \overline { a } ^ { 2 } \overline { b } ^ { 2 } \sqrt { \overline { b } } } \sum _ { s = 1 } ^ { p } \gamma _ { 3 } ( s ) s ( 1 - \frac { 3 \overline { c } } { \overline { a } \overline { b } } ) ^ { s - 1 } \left[\bar{a} \bar{b}\left(\overline{\mathbf{A}}_{k l}^{-1} \overline{\mathbf{J}}_{11} \overline{\mathbf{A}}_{k l}^{-1} \overline{\mathbf{J}}_{g} \overline{\mathbf{A}}_{k l}^{-1}\right)_{\alpha \beta}\right.\right. \\
& \left.\left.+\bar{a} \bar{b}\left(\overline{\mathbf{A}}_{k l}^{-1} \overline{\mathbf{J}}_{g} \overline{\mathbf{A}}_{k l}^{-1} \overline{\mathbf{J}}_{11} \overline{\mathbf{A}}_{k l}^{-1}\right)_{\alpha \beta}-\bar{a} \bar{c}\left(\overline{\mathbf{A}}_{k l}^{-1} \overline{\mathbf{J}}_{g} \overline{\mathbf{A}}_{k l}^{-1}\right)_{\alpha \beta}-\bar{b} \bar{c}\left(\overline{\mathbf{A}}_{k l}^{-1} \overline{\mathbf{J}}_{11} \overline{\mathbf{A}}_{k l}^{-1}\right)_{\alpha \beta}\right]\right\} \\
& =-\frac{1}{2} \overline{\mathbf{B}}_{\alpha}^{\beta} \overline{\mathbf{C}}_{\rho}^{\gamma}\left(\partial_{\rho \gamma}\left\langle\phi_{k}\left|r_{1}^{-q} \frac{1}{r_{g}}\right| \phi_{l}\right\rangle\right)\left[\frac{m_{k l}-q}{\bar{a}}\left(\overline{\mathbf{A}}_{k l}^{-1} \overline{\mathbf{J}}_{11} \overline{\mathbf{A}}_{k l}^{-1}\right)_{\alpha \beta}+\left(\overline{\mathbf{A}}_{k l}^{-1}\right)_{\alpha \beta}-\frac{1}{\bar{b}}\left(\overline{\mathbf{A}}_{k l}^{-1} \overline{\mathbf{J}}_{g} \overline{\mathbf{A}}_{k l}^{-1}\right)_{\alpha \beta}\right]-\frac{1}{2}\left\langle\phi_{k}\left|r_{1}^{-q} \frac{1}{r_{g}}\right| \phi_{l}\right\rangle \overline{\mathbf{B}}_{\alpha}^{\beta} \overline{\mathbf{C}}_{\rho}^{\gamma} \partial_{\rho \gamma} \\
& \times\left\{\frac{m_{k l}-q}{\bar{a}}\left(\overline{\mathbf{A}}_{k l}^{-1} \overline{\mathbf{J}}_{11} \overline{\mathbf{A}}_{k l}^{-1}\right)_{\alpha \beta}+\left(\overline{\mathbf{A}}_{k l}^{-1}\right)_{\alpha \beta}-\frac{1}{\bar{b}}\left(\overline{\mathbf{A}}_{k l}^{-1} \overline{\mathbf{J}}_{g} \overline{\mathbf{A}}_{k l}^{-1}\right)_{\alpha \beta}\right\}+\frac{3 \sqrt{3} \gamma_{2}(p)}{\bar{a}^{2} \bar{b}^{2} \sqrt{\bar{b}}} \sum_{s=1}^{p} \gamma_{3}(s) s\left(1-\frac{3 \bar{c}}{\bar{a} \bar{b}}\right)^{s-1} \overline{\mathbf{B}}_{\alpha}^{\beta} \overline{\mathbf{C}}_{\rho}^{\gamma}\left(\partial_{\rho \gamma}\left\langle\phi_{k}\left|r_{1}^{-q}\right| \phi_{l}\right\rangle\right) \\
& \times\left[\bar{a} \bar{b}\left(\overline{\mathbf{A}}_{k l}^{-1} \overline{\mathbf{J}}_{11} \overline{\mathbf{A}}_{k l}^{-1} \overline{\mathbf{J}}_{g} \overline{\mathbf{A}}_{k l}^{-1}\right)_{\alpha \beta}+\bar{a} \bar{b}\left(\overline{\mathbf{A}}_{k l}^{-1} \overline{\mathbf{J}}_{g} \overline{\mathbf{A}}_{k l}^{-1} \overline{\mathbf{J}}_{11} \overline{\mathbf{A}}_{k l}^{-1}\right)_{\alpha \beta}-\bar{a} \bar{c}\left(\overline{\mathbf{A}}_{k l}^{-1} \overline{\mathbf{J}}_{g} \overline{\mathbf{A}}_{k l}^{-1}\right)_{\alpha \beta}-\bar{b} \bar{c}\left(\overline{\mathbf{A}}_{k l}^{-1} \overline{\mathbf{J}}_{11} \overline{\mathbf{A}}_{k l}^{-1}\right)_{\alpha \beta}\right] \\
& \times\left\langle\phi_{k}\left|r_{1}^{-q}\right| \phi_{l}\right\rangle 3 \sqrt{3} \gamma_{2}(p) \sum_{s=1}^{p} \gamma_{3}(s) s\left(1-\frac{3 \bar{c}}{\bar{a} \bar{b}}\right)^{s-1} \overline{\mathbf{B}}_{\alpha}^{\beta} \overline{\mathbf{C}}_{\rho}^{\gamma}\left(\partial_{\rho \gamma}-\frac{1}{\bar{a}^{2} \bar{b}^{2} \sqrt{\bar{b}}}\right)\left[\bar{a} \bar{b}\left(\overline{\mathbf{A}}_{k l}^{-1} \overline{\mathbf{J}}_{11} \overline{\mathbf{A}}_{k l}^{-1} \overline{\mathbf{J}}_{g} \overline{\mathbf{A}}_{k l}^{-1}\right)_{\alpha \beta}\right. \\
& \left.+\bar{a} \bar{b}\left(\overline{\mathbf{A}}_{k l}^{-1} \overline{\mathbf{J}}_{g} \overline{\mathbf{A}}_{k l}^{-1} \overline{\mathbf{J}}_{11} \overline{\mathbf{A}}_{k l}^{-1}\right)_{\alpha \beta}-\bar{a} \bar{c}\left(\overline{\mathbf{A}}_{k l}^{-1} \overline{\mathbf{J}}_{g} \overline{\mathbf{A}}_{k l}^{-1}\right)_{\alpha \beta}-\bar{b} \bar{c}\left(\overline{\mathbf{A}}_{k l}^{-1} \overline{\mathbf{J}}_{11} \overline{\mathbf{A}}_{k l}^{-1}\right)_{\alpha \beta}\right]-3\left\langle\phi_{k}\left|r_{1}^{-q}\right| \phi_{l}\right\rangle \frac{3 \sqrt{3} \gamma_{2}(p)}{\bar{a}^{2} \bar{b}^{2} \sqrt{\bar{b}}} \\
& \times \sum_{s=1}^{p} \gamma_{3}(s) s(s-1)\left(1-\frac{3 \bar{c}}{\bar{a} \bar{b}}\right)^{s-2} \overline{\mathbf{B}}_{\alpha}^{\beta} \overline{\mathbf{C}}_{\rho}^{\gamma}\left(\partial_{\rho \gamma} \frac{\bar{c}}{\bar{a} \bar{b}}\right)\left[\bar{a} \bar{b}\left(\overline{\mathbf{A}}_{k l}^{-1} \overline{\mathbf{J}}_{11} \overline{\mathbf{A}}_{k l}^{-1} \overline{\mathbf{J}}_{g} \overline{\mathbf{A}}_{k l}^{-1}\right)_{\alpha \beta}+\bar{a} \bar{b}\left(\overline{\mathbf{A}}_{k l}^{-1} \overline{\mathbf{J}}_{g} \overline{\mathbf{A}}_{k l}^{-1} \overline{\mathbf{J}}_{11} \overline{\mathbf{A}}_{k l}^{-1}\right)_{\alpha \beta}\right. \\
& \left.-\bar{a} \bar{c}\left(\overline{\mathbf{A}}_{k l}^{-1} \overline{\mathbf{J}}_{g} \overline{\mathbf{A}}_{k l}^{-1}\right)_{\alpha \beta}-\bar{b} \bar{c}\left(\overline{\mathbf{A}}_{k l}^{-1} \overline{\mathbf{J}}_{11} \overline{\mathbf{A}}_{k l}^{-1}\right)_{\alpha \beta}\right]+\left\langle\phi_{k}\left|r_{1}^{-q}\right| \phi_{l}\right\rangle \frac{3 \sqrt{3} \gamma_{2}(p)}{\bar{a}^{2} \bar{b}^{2} \sqrt{\bar{b}}} \\
& \times \sum_{s=1}^{p} \gamma_{3}(s) s\left(1-\frac{3 \bar{c}}{\bar{a} \bar{b}}\right)^{s-1} \overline{\mathbf{B}}_{\alpha}^{\beta} \overline{\mathbf{C}}_{\rho}^{\gamma} \partial_{\rho \gamma}\left\{\bar{a} \bar{b}\left(\overline{\mathbf{A}}_{k l}^{-1} \overline{\mathbf{J}}_{11} \overline{\mathbf{A}}_{k l}^{-1} \overline{\mathbf{J}}_{g} \overline{\mathbf{A}}_{k l}^{-1}\right)_{\alpha \beta}\right. \\
& \left.+\bar{a} \bar{b}\left(\overline{\mathbf{A}}_{k l}^{-1} \overline{\mathbf{J}}_{g} \overline{\mathbf{A}}_{k l}^{-1} \overline{\mathbf{J}}_{11} \overline{\mathbf{A}}_{k l}^{-1}\right)_{\alpha \beta}-\bar{a} \bar{c}\left(\overline{\mathbf{A}}_{k l}^{-1} \overline{\mathbf{J}}_{g} \overline{\mathbf{A}}_{k l}^{-1}\right)_{\alpha \beta}-\bar{b} \bar{c}\left(\overline{\mathbf{A}}_{k l}^{-1} \overline{\mathbf{J}}_{11} \overline{\mathbf{A}}_{k l}^{-1}\right)_{\alpha \beta}\right\} .
\end{aligned}
$$

To conclude the task we still need to determine the following derivatives:

(1) $\partial_{\rho \gamma}\left\langle\phi_{k}\left|r_{1}^{-q} 1 / r_{g}\right| \phi_{l}\right\rangle$,

(2) $\partial_{\rho \gamma}(\bar{a})^{-1}$,

(3) $\partial_{\rho \gamma}\left(\overline{\mathbf{A}}_{k l}^{-1} \overline{\mathbf{J}}_{11} \overline{\mathbf{A}}_{k l}^{-1}\right)_{\alpha \beta}$,

(4) $\partial_{\rho \gamma}\left(\overline{\mathbf{A}}_{k l}^{-1}\right)_{\alpha \beta}$,

(5) $\partial_{\rho \gamma}(\bar{b})^{-1}$,

(6) $\partial_{\rho \gamma}\left(\overline{\mathbf{A}}_{k l}^{-1} \overline{\mathbf{J}}_{g} \overline{\mathbf{A}}_{k l}^{-1}\right)_{\alpha \beta}$,

(7) $\partial_{\rho \gamma}\left\langle\phi_{k}\left|r_{1}^{-q}\right| \phi_{l}\right\rangle$,

(8) $\partial_{\rho \gamma}\left(\bar{a}^{2} \bar{b}^{2} \sqrt{b}\right)^{-1}$,

(9) $\partial_{\rho \gamma} 3 \bar{c}(\bar{a} \bar{b})^{-1}$, 
(10)

$$
\partial_{\rho \gamma}\left[\bar{a} \bar{b}\left(\overline{\mathbf{A}}_{k l}^{-1} \overline{\mathbf{J}}_{11} \overline{\mathbf{A}}_{k l}^{-1} \overline{\mathbf{J}}_{g} \overline{\mathbf{A}}_{k l}^{-1}\right)_{\alpha \beta}+\bar{a} \bar{b}\left(\overline{\mathbf{A}}_{k l}^{-1} \overline{\mathbf{J}}_{g} \overline{\mathbf{A}}_{k l}^{-1} \overline{\mathbf{J}}_{11} \overline{\mathbf{A}}_{k l}^{-1}\right)_{\alpha \beta}-\overline{a c}\left(\overline{\mathbf{A}}_{k l}^{-1} \overline{\mathbf{J}}_{g} \overline{\mathbf{A}}_{k l}^{-1}\right)_{\alpha \beta}-\bar{b} \bar{c}\left(\overline{\mathbf{A}}_{k l}^{-1} \overline{\mathbf{J}}_{11} \overline{\mathbf{A}}_{k l}^{-1}\right)_{\alpha \beta}\right] .
$$

We now evaluate derivatives in the following:

ad.1. $\quad \partial_{\rho \gamma}\left\langle\phi_{K}\left|r_{1}^{-q} 1 / r_{g}\right| \phi_{L}\right\rangle=-\frac{1}{2}\left\langle\phi_{k}\left|r_{1}^{-q} 1 / r_{g}\right| \phi_{l}\right\rangle\left[m_{k l}-q / \bar{a}\left(\overline{\mathbf{A}}_{k l}^{-1} \overline{\mathbf{J}}_{11} \overline{\mathbf{A}}_{k l}^{-1}\right)_{\rho \gamma}+\left(\overline{\mathbf{A}}_{k l}^{-1}\right)_{\rho \gamma}-1 / \bar{b}\left(\overline{\mathbf{A}}_{k l}^{-1} \overline{\mathbf{J}}_{g} \overline{\mathbf{A}}_{k l}^{-1}\right)_{\rho \gamma}\right]$

$$
\begin{aligned}
& +\left\langle\phi_{k}\left|r_{1}^{-q}\right| \phi_{l}\right\rangle 3 \sqrt{3} \gamma_{2}(p) / \\
& \bar{a}^{2} \bar{b}^{2} \sqrt{\bar{b}} \sum_{s=1}^{p} \gamma_{3}(s) s(1-3 \bar{c} / \bar{a} \bar{b})^{s-1}\left[\bar{a} \bar{b}\left(\overline{\mathbf{A}}_{k l}^{-1} \overline{\mathbf{J}}_{11} \overline{\mathbf{A}}_{k l}^{-1} \overline{\mathbf{J}}_{g} \overline{\mathbf{A}}_{k l}^{-1}\right)_{\rho \gamma}+\bar{a} \bar{b}\left(\overline{\mathbf{A}}_{k l}^{-1} \overline{\mathbf{J}}_{g} \overline{\mathbf{A}}_{k l}^{-1} \overline{\mathbf{J}}_{11} \overline{\mathbf{A}}_{k l}^{-1}\right)_{\rho \gamma}\right. \\
& \left.-\overline{a c}\left(\overline{\mathbf{A}}_{k l}^{-1} \overline{\mathbf{J}}_{g} \overline{\mathbf{A}}_{k l}^{-1}\right)_{\rho \gamma}-\bar{b} \bar{c}\left(\overline{\mathbf{A}}_{k l}^{-1} \overline{\mathbf{J}}_{11} \overline{\mathbf{A}}_{k l}^{-1}\right)_{\rho \gamma}\right],
\end{aligned}
$$

ad.2. $\partial_{\rho \gamma} 1 / \bar{a}=-\bar{a}^{-2} \partial_{\rho \gamma} \bar{a}=\bar{a}^{-2}\left(\overline{\mathbf{A}}_{k l}^{-1} \overline{\mathbf{J}}_{11} \overline{\mathbf{A}}_{k l}^{-1}\right)_{\rho \gamma}$,

ad.3. $\quad \partial_{\rho \gamma}\left(\overline{\mathbf{A}}_{k l}^{-1} \overline{\mathbf{J}}_{11} \overline{\mathbf{A}}_{k l}^{-1}\right)_{\alpha \beta}=-\left[\left(\overline{\mathbf{A}}_{k l}^{-1}\right)_{\alpha \rho}\left(\overline{\mathbf{A}}_{k l}^{-1} \overline{\mathbf{J}}_{11} \overline{\mathbf{A}}_{k l}^{-1}\right)_{\gamma \beta}+\left(\overline{\mathbf{A}}_{k l}^{-1} \overline{\mathbf{J}}_{11} \overline{\mathbf{A}}_{k l}^{-1}\right)_{\alpha \rho}\left(\overline{\mathbf{A}}_{k l}^{-1}\right)_{\gamma \beta}\right]$,

ad.4. $\quad \partial_{\rho \gamma}\left(\overline{\mathbf{A}}_{k l}^{-1}\right)_{\alpha \beta}=-\left(\overline{\mathbf{A}}_{k l}\right)_{\alpha \rho}^{-1}\left(\overline{\mathbf{A}}_{k l}\right)_{\beta \gamma}^{-1}$,

ad.5. $\partial_{\rho \gamma} 1 / \bar{b}=-\bar{b}^{-2} \partial_{\rho \gamma} \bar{b}=\bar{b}^{-2}\left(\overline{\mathbf{A}}_{k l}^{-1} \overline{\mathbf{J}}_{g} \overline{\mathbf{A}}_{k l}^{-1}\right)_{\rho \gamma}$,

ad.6. $\quad \partial_{\rho \gamma}\left(\overline{\mathbf{A}}_{k l}^{-1} \overline{\mathbf{J}}_{g} \overline{\mathbf{A}}_{k l}^{-1}\right)_{\alpha \beta}=-\left[\left(\overline{\mathbf{A}}_{k l}^{-1}\right)_{\alpha \rho}\left(\overline{\mathbf{A}}_{k l}^{-1} \overline{\mathbf{J}}_{g} \overline{\mathbf{A}}_{k l}^{-1}\right)_{\gamma \beta}+\left(\overline{\mathbf{A}}_{k l}^{-1} \overline{\mathbf{J}}_{g} \overline{\mathbf{A}}_{k l}^{-1}\right)_{\alpha \rho}\left(\overline{\mathbf{A}}_{k l}^{-1}\right)_{\gamma \beta}\right]$,

ad.7. $\quad \partial_{\rho \gamma}\left\langle\phi_{k}\left|r_{1}^{-q}\right| \phi_{l}\right\rangle=-\left\langle\phi_{k}\left|r_{1}^{-q}\right| \phi_{l}\right\rangle\left[m_{m k}-q / 2 \bar{a}\left(\overline{\mathbf{A}}_{k l}^{-1} \overline{\mathbf{J}}_{11} \overline{\mathbf{A}}_{k l}^{-1}\right)_{\rho \gamma}+\frac{1}{2}\left(\overline{\mathbf{A}}_{k l}^{-1}\right)_{\rho \gamma}\right]$,

ad.8. $\quad \partial_{\rho \gamma}\left(\bar{b}^{-5 / 2} \bar{a}^{-2}\right)=1 / \sqrt{\bar{b}} 1 / \bar{a}^{2} \bar{b}^{2}\left[\frac{5}{2} 1 / \bar{b}\left(\overline{\mathbf{A}}_{k l}^{-1} \overline{\mathbf{J}}_{g} \overline{\mathbf{A}}_{k l}^{-1}\right)_{\rho \gamma}+21 / \bar{a}\left(\overline{\mathbf{A}}_{k l}^{-1} \overline{\mathbf{J}}_{11} \overline{\mathbf{A}}_{k l}^{-1}\right)_{\rho \gamma}\right]$,

ad.9. $\quad \partial_{\rho \gamma}(\bar{c} / \bar{a} \bar{b})=-1 / \bar{a}^{2} \bar{b}^{2}\left[+\bar{a} \bar{b}\left(\overline{\mathbf{A}}_{k l}^{-1} \overline{\mathbf{J}}_{11} \overline{\mathbf{A}}_{k l}^{-1} \overline{\mathbf{J}}_{g} \overline{\mathbf{A}}_{k l}^{-1}\right)_{\rho \gamma}+\bar{a} \bar{b}\left(\overline{\mathbf{A}}_{k l}^{-1} \overline{\mathbf{J}}_{g} \overline{\mathbf{A}}_{k l}^{-1} \overline{\mathbf{J}}_{11} \overline{\mathbf{A}}_{k l}^{-1}\right)_{\rho \gamma}-\bar{c} \bar{b}\left(\overline{\mathbf{A}}_{k l}^{-1} \overline{\mathbf{J}}_{11} \overline{\mathbf{A}}_{k l}^{-1}\right)_{\rho \gamma}-\overline{c a}\left(\overline{\mathbf{A}}_{k l}^{-1} \overline{\mathbf{J}}_{g} \overline{\mathbf{A}}_{k l}^{-1}\right)_{\rho \gamma}\right]$,

ad.10. $\quad \partial_{\rho \gamma}\left[\bar{a} \bar{b}\left(\overline{\mathbf{A}}_{k l}^{-1} \overline{\mathbf{J}}_{11} \overline{\mathbf{A}}_{k l}^{-1} \overline{\mathbf{J}}_{g} \overline{\mathbf{A}}_{k l}^{-1}\right)_{\alpha \beta}+\bar{a} \bar{b}\left(\overline{\mathbf{A}}_{k l}^{-1} \overline{\mathbf{J}}_{g} \overline{\mathbf{A}}_{k l}^{-1} \overline{\mathbf{J}}_{11} \overline{\mathbf{A}}_{k l}^{-1}\right)_{\alpha \beta}-\overline{a c}\left(\overline{\mathbf{A}}_{k l}^{-1} \overline{\mathbf{J}}_{g} \overline{\mathbf{A}}_{k l}^{-1}\right)_{\alpha \beta}-\bar{b} \bar{c}\left(\overline{\mathbf{A}}_{k l}^{-1} \overline{\mathbf{J}}_{11} \overline{\mathbf{A}}_{k l}^{-1}\right)_{\alpha \beta}\right]$

$$
\begin{aligned}
= & \left.\left(\partial_{\rho \gamma} \bar{a} \bar{b}\right)\left(\left(\overline{\mathbf{A}}_{k l}^{-1} \overline{\mathbf{J}}_{11} \overline{\mathbf{A}}_{k l}^{-1} \overline{\mathbf{J}}_{g} \overline{\mathbf{A}}_{k l}^{-1}\right)_{\alpha \beta}+\left(\overline{\mathbf{A}}_{k l}^{-1} \overline{\mathbf{J}}_{g} \overline{\mathbf{A}}_{k l}^{-1} \overline{\mathbf{J}}_{11} \overline{\mathbf{A}}_{k l}^{-1}\right)_{\alpha \beta}\right)+\bar{a} \bar{b} \partial_{\rho \gamma}\left(\overline{\mathbf{A}}_{k l}^{-1} \overline{\mathbf{J}}_{11} \overline{\mathbf{A}}_{k l}^{-1} \overline{\mathbf{J}}_{g} \overline{\mathbf{A}}_{k l}^{-1}\right)_{\alpha \beta}+\left(\overline{\mathbf{A}}_{k l}^{-1} \overline{\mathbf{J}}_{g} \overline{\mathbf{A}}_{k l}^{-1} \overline{\mathbf{J}}_{11} \overline{\mathbf{A}}_{k l}^{-1}\right)_{\alpha \beta}\right\}-\left(\partial_{\rho \gamma} \overline{c c}\right) \\
& \times\left(\overline{\mathbf{A}}_{k l}^{-1} \overline{\mathbf{J}}_{g} \overline{\mathbf{A}}_{k l}^{-1}\right)_{\alpha \beta}-\left(\partial_{\rho \gamma} \bar{b} \bar{c}\right)\left(\overline{\mathbf{A}}_{k l}^{-1} \overline{\mathbf{J}}_{11} \overline{\mathbf{A}}_{k l}^{-1}\right)_{\alpha \beta}-\overline{a c} \partial_{\rho \gamma}\left(\overline{\mathbf{A}}_{k l}^{-1} \overline{\mathbf{J}}_{g} \overline{\mathbf{A}}_{k l}^{-1}\right)_{\alpha \beta}-\bar{b} \bar{c} \partial_{\rho \gamma}\left(\overline{\mathbf{A}}_{k l}^{-1} \overline{\mathbf{J}}_{11} \overline{\mathbf{A}}_{k l}^{-1}\right)_{\alpha \beta},
\end{aligned}
$$

where

$$
\begin{aligned}
& \left(\partial_{\rho \gamma} \bar{a} \bar{b}\right)=-\bar{b}\left(\overline{\mathbf{A}}_{k l}^{-1} \overline{\mathbf{J}}_{11} \overline{\mathbf{A}}_{k l}^{-1}\right)_{\rho \gamma}-\bar{a}\left(\overline{\mathbf{A}}_{k l}^{-1} \overline{\mathbf{J}}_{g} \overline{\mathbf{A}}_{k l}^{-1}\right)_{\rho \gamma} \\
& \left(\partial_{\rho \gamma} \bar{a} \bar{c}\right)=-\bar{c}\left(\overline{\mathbf{A}}_{k l}^{-1} \overline{\mathbf{J}}_{11} \overline{\mathbf{A}}_{k l}^{-1}\right)_{\rho \gamma}-\bar{a}\left[\left(\overline{\mathbf{A}}_{k l}^{-1} \overline{\mathbf{J}}_{11} \overline{\mathbf{A}}_{k l}^{-1} \overline{\mathbf{J}}_{g} \overline{\mathbf{A}}_{k l}^{-1}\right)_{\rho \gamma}+\left(\overline{\mathbf{A}}_{k l}^{-1} \overline{\mathbf{J}}_{g} \overline{\mathbf{A}}_{k l}^{-1} \overline{\mathbf{J}}_{11} \overline{\mathbf{A}}_{k l}^{-1}\right)_{\rho \gamma}\right] \\
& \left(\partial_{\rho \gamma} \bar{b} \bar{c}\right)=-\bar{c}\left(\overline{\mathbf{A}}_{k l}^{-1} \overline{\mathbf{J}}_{g} \overline{\mathbf{A}}_{k l}^{-1}\right)_{\rho \gamma}-\bar{b}\left[\left(\overline{\mathbf{A}}_{k l}^{-1} \overline{\mathbf{J}}_{11} \overline{\mathbf{A}}_{k l}^{-1} \overline{\mathbf{J}}_{g} \overline{\mathbf{A}}_{k l}^{-1}\right)_{\rho \gamma}+\left(\overline{\mathbf{A}}_{k l}^{-1} \overline{\mathbf{J}}_{g} \overline{\mathbf{A}}_{k l}^{-1} \overline{\mathbf{J}}_{11} \overline{\mathbf{A}}_{k l}^{-1}\right)_{\rho \gamma}\right] \\
& \partial_{\rho \gamma}\left(\overline{\mathbf{A}}_{k l}^{-1} \overline{\mathbf{J}}_{11} \overline{\mathbf{A}}_{k l}^{-1} \overline{\mathbf{J}}_{g} \overline{\mathbf{A}}_{k l}^{-1}\right)_{\alpha \beta}=-\left[\left(\overline{\mathbf{A}}_{k l}^{-1}\right)_{\alpha \rho}\left(\overline{\mathbf{A}}_{k l}^{-1} \overline{\mathbf{J}}_{11} \overline{\mathbf{A}}_{k l}^{-1} \overline{\mathbf{J}}_{g} \overline{\mathbf{A}}_{k l}^{-1}\right)_{\gamma \beta}+\left(\overline{\mathbf{A}}_{k l}^{-1} \overline{\mathbf{J}}_{11} \overline{\mathbf{A}}_{k l}^{-1}\right)_{\alpha \rho}\left(\overline{\mathbf{A}}_{k l}^{-1} \overline{\mathbf{J}}_{g} \overline{\mathbf{A}}_{k l}^{-1}\right)_{\gamma \beta}+\left(\overline{\mathbf{A}}_{k l}^{-1} \overline{\mathbf{J}}_{11} \overline{\mathbf{A}}_{k l}^{-1} \overline{\mathbf{J}}_{g} \overline{\mathbf{A}}_{k l}^{-1}\right)_{\alpha \rho}+\left(\overline{\mathbf{A}}_{k l}^{-1}\right)_{\gamma \beta}\right] \\
& \partial_{\rho \gamma}\left(\overline{\mathbf{A}}_{k l}^{-1} \overline{\mathbf{J}}_{g} \overline{\mathbf{A}}_{k l}^{-1} \overline{\mathbf{J}}_{11} \overline{\mathbf{A}}_{k l}^{-1}\right)_{\alpha \beta}=-\left[\left(\overline{\mathbf{A}}_{k l}^{-1}\right)_{\alpha \rho}\left(\overline{\mathbf{A}}_{k l}^{-1} \overline{\mathbf{J}}_{g} \overline{\mathbf{A}}_{k l}^{-1} \overline{\mathbf{J}}_{11} \overline{\mathbf{A}}_{k l}^{-1}\right)_{\gamma \beta}+\left(\overline{\mathbf{A}}_{k l}^{-1} \overline{\mathbf{J}}_{g} \overline{\mathbf{A}}_{k l}^{-1}\right)_{\alpha \rho}\left(\overline{\mathbf{A}}_{k l}^{-1} \overline{\mathbf{J}}_{11} \overline{\mathbf{A}}_{k l}^{-1}\right)_{\gamma \beta}+\left(\overline{\mathbf{A}}_{k l}^{-1} \overline{\mathbf{J}}_{g} \overline{\mathbf{A}}_{k l}^{-1} \overline{\mathbf{J}}_{11} \overline{\mathbf{A}}_{k l}^{-1}\right)_{\alpha \rho}+\left(\overline{\mathbf{A}}_{k l}^{-1}\right)_{\gamma \beta}\right] \\
& \partial_{\rho \gamma}\left(\overline{\mathbf{A}}_{k l}^{-1} \overline{\mathbf{J}}_{g} \overline{\mathbf{A}}_{k l}^{-1}\right)_{\alpha \beta}=-\left[\left(\overline{\mathbf{A}}_{k l}^{-1}\right)_{\alpha \rho}\left(\overline{\mathbf{A}}_{k l}^{-1} \overline{\mathbf{J}}_{g} \overline{\mathbf{A}}_{k l}^{-1}\right)_{\gamma \beta}+\left(\overline{\mathbf{A}}_{k l}^{-1} \overline{\mathbf{J}}_{g} \overline{\mathbf{A}}_{k l}^{-1}\right)_{\alpha \rho}\left(\overline{\mathbf{A}}_{k l}^{-1}\right)_{\gamma \beta}\right] \\
& \partial_{\rho \gamma}\left(\overline{\mathbf{A}}_{k l}^{-1} \overline{\mathbf{J}}_{11} \overline{\mathbf{A}}_{k l}^{-1}\right)_{\alpha \beta}=-\left[\left(\overline{\mathbf{A}}_{k l}^{-1}\right)_{\alpha \rho}\left(\overline{\mathbf{A}}_{k l}^{-1} \overline{\mathbf{J}}_{11} \overline{\mathbf{A}}_{k l}^{-1}\right)_{\gamma \beta}+\left(\overline{\mathbf{A}}_{k l}^{-1} \overline{\mathbf{J}}_{11} \overline{\mathbf{A}}_{k l}^{-1}\right)_{\alpha \rho}\left(\overline{\mathbf{A}}_{k l}^{-1}\right)_{\gamma \beta}\right] .
\end{aligned}
$$

Using the above we can write the expressions appearing in Eq. (B1) as 
- $\overline{\mathbf{B}}^{\alpha \beta} \overline{\mathbf{C}}^{\rho \gamma}\left(\partial_{\rho \gamma}\left\langle\phi_{k}\left|r_{1}^{-q} \frac{1}{r_{g}}\right| \phi_{l}\right\rangle\right)\left[\frac{m_{k l}-q}{\bar{a}}\left(\overline{\mathbf{A}}_{k l}^{-1} \overline{\mathbf{J}}_{11} \overline{\mathbf{A}}_{k l}^{-1}\right)_{\alpha \beta}+\left(\overline{\mathbf{A}}_{k l}^{-1}\right)_{\alpha \beta}-\frac{1}{\bar{b}}\left(\overline{\mathbf{A}}_{k l}^{-1} \overline{\mathbf{J}}_{g} \overline{\mathbf{A}}_{k l}^{-1}\right)_{\alpha \beta}\right]$

$$
\begin{aligned}
= & -\frac{1}{2}\left\langle\phi_{k}\left|r_{1}^{-q} \frac{1}{r_{g}}\right| \phi_{l}\right\rangle\left[\frac{m_{k l}-q}{\bar{a}} \operatorname{tr}\left[\overline{\mathbf{A}}_{k l}^{-1} \overline{\mathbf{J}}_{11} \overline{\mathbf{A}}_{k l}^{-1} \overline{\mathbf{C}}\right]+\operatorname{tr}\left[\overline{\mathbf{A}}_{k l}^{-1} \overline{\mathbf{C}}^{-1}-\frac{1}{\bar{b}} \operatorname{tr}\left[\overline{\mathbf{A}}_{k l}^{-1} \overline{\mathbf{J}}_{g} \overline{\mathbf{A}}_{k l}^{-1} \overline{\mathbf{C}}\right]\right]\right. \\
& \times\left[\frac{m_{k l}-q}{\bar{a}} \operatorname{tr}\left[\overline{\mathbf{A}}_{k l}^{-1} \overline{\mathbf{J}}_{11} \overline{\mathbf{A}}_{k l}^{-1} \overline{\mathbf{B}}\right]+\operatorname{tr}\left[\overline{\mathbf{A}}_{k l}^{-1} \overline{\mathbf{B}}\right]-\frac{1}{\bar{b}} \operatorname{tr}\left[\overline{\mathbf{A}}_{k l}^{-1} \overline{\mathbf{J}}_{g} \overline{\mathbf{A}}_{k l}^{-1} \overline{\mathbf{B}}\right]\right]+\left\langle\phi_{k}\left|r_{1}^{-q}\right| \phi_{l}\right\rangle \frac{3 \sqrt{3} \gamma_{2}(p)}{\bar{a}^{2} \bar{b}^{2} \sqrt{\bar{b}}} \sum_{s=1}^{p} \gamma_{3}(s) s\left(1-\frac{3 \bar{c}}{\bar{a} \bar{b}}\right)^{s-1}
\end{aligned}
$$$$
\times\left[\bar{a} \bar{b}\left(\operatorname{tr}\left[\overline{\mathbf{A}}_{k l}^{-1} \overline{\mathbf{J}}_{g} \overline{\mathbf{A}}_{k l}^{-1} \overline{\mathbf{J}}_{11} \overline{\mathbf{A}}_{k l}^{-1} \overline{\mathbf{C}}^{\prime}\right]+\operatorname{tr}\left[\overline{\mathbf{A}}_{k l}^{-1} \overline{\mathbf{J}}_{11} \overline{\mathbf{A}}_{k l}^{-1} \overline{\mathbf{J}}_{g} \overline{\mathbf{A}}_{k l}^{-1} \overline{\mathbf{C}}\right]\right)-\bar{a} \bar{c} \operatorname{tr}\left[\overline{\mathbf{A}}_{k l}^{-1} \overline{\mathbf{J}}_{g} \overline{\mathbf{A}}_{k l}^{-1} \overline{\mathbf{C}}\right]-\bar{b} \bar{c} \operatorname{tr}\left[\overline{\mathbf{A}}_{k l}^{-1} \overline{\mathbf{J}}_{11} \overline{\mathbf{A}}_{k l}^{-1} \overline{\mathbf{C}}\right]\right]
$$$$
\times\left[\frac{m_{k l}-q}{\bar{a}} \operatorname{tr}\left[\overline{\mathbf{A}}_{k l}^{-1} \overline{\mathbf{J}}_{11} \overline{\mathbf{A}}_{k l}^{-1} \overline{\mathbf{B}}\right]+\operatorname{tr}\left[\overline{\mathbf{A}}_{k l}^{-1} \overline{\mathbf{B}}\right]-\frac{1}{\bar{b}} \operatorname{tr}\left[\overline{\mathbf{A}}_{k l}^{-1} \overline{\mathbf{J}}_{g} \overline{\mathbf{A}}_{k l}^{-1} \overline{\mathbf{B}}\right]\right],
$$

- $\overline{\mathbf{B}}^{\alpha \beta} \overline{\mathbf{C}}^{\rho \gamma} \partial_{\rho \gamma}\left[\frac{m_{k l}-q}{\bar{a}}\left(\overline{\mathbf{A}}_{k l}^{-1} \overline{\mathbf{J}}_{11} \overline{\mathbf{A}}_{k l}^{-1}\right)_{\alpha \beta}+\left(\overline{\mathbf{A}}_{k l}^{-1}\right)_{\alpha \beta}-\frac{1}{\bar{b}}\left(\overline{\mathbf{A}}_{k l}^{-1} \overline{\mathbf{J}}_{g} \overline{\mathbf{A}}_{k l}^{-1}\right)_{\alpha \beta}\right]$

$$
\begin{aligned}
= & \frac{\left(m_{k l}-q\right)}{\bar{a}}\left(\frac{1}{a} \operatorname{tr}\left[\overline{\mathbf{A}}_{k l}^{-1} \overline{\mathbf{J}}_{11} \overline{\mathbf{A}}_{k l}^{-1} \overline{\mathbf{C}}\right] \operatorname{tr}\left[\overline{\mathbf{A}}_{k l}^{-1} \overline{\mathbf{J}}_{11} \overline{\mathbf{A}}_{k l}^{-1} \overline{\mathbf{B}}\right]-\operatorname{tr}\left[\overline{\mathbf{A}}_{k l}^{-1} \overline{\mathbf{B}}_{k l}^{-1} \overline{\mathbf{J}}_{11} \overline{\mathbf{A}}_{k l}^{-1} \overline{\mathbf{C}}^{\prime}\right]-\operatorname{tr}\left[\overline{\mathbf{A}}_{k l}^{-1} \overline{\mathbf{J}}_{11} \overline{\mathbf{A}}_{k l}^{-1} \overline{\mathbf{B}} \overline{\mathbf{A}}_{k l}^{-1} \overline{\mathbf{C}}^{\prime}\right]\right) \\
& -\operatorname{tr}\left[\overline{\mathbf{A}}_{k l}^{-1} \overline{\mathbf{B}} \overline{\mathbf{A}}_{k l}^{-1} \overline{\mathbf{C}}^{\prime}\right]+\frac{1}{\bar{b}}\left(-\frac{1}{\bar{b}} \operatorname{tr}\left[\overline{\mathbf{A}}_{k l}^{-1} \overline{\mathbf{J}}_{g} \overline{\mathbf{A}}_{k l}^{-1} \overline{\mathbf{C}}_{\ln }\right] \operatorname{tr}\left[\overline{\mathbf{A}}_{k l}^{-1} \overline{\mathbf{J}}_{g} \overline{\mathbf{A}}_{k l}^{-1} \overline{\mathbf{B}}\right]+\operatorname{tr}\left[\overline{\mathbf{C}}_{k l}^{-1} \overline{\mathbf{J}}_{g} \overline{\mathbf{A}}_{k l}^{-1} \overline{\mathbf{B}}^{\prime} \overline{\mathbf{A}}_{k l}^{-1}\right]+\operatorname{tr}\left[\overline{\mathbf{B}}^{\prime} \overline{\mathbf{A}}_{k l}^{-1} \overline{\mathbf{J}}_{g} \overline{\mathbf{A}}_{k l}^{-1} \overline{\mathbf{C}}_{k l}^{-1} \overline{\mathbf{A}}_{k l}^{-1}\right),\right.
\end{aligned}
$$

- $\quad \overline{\mathbf{B}}^{\alpha \beta} \overline{\mathbf{C}}^{\rho \gamma}\left(\partial_{\rho \gamma}\left\langle\phi_{k}\left|r_{1}^{-q}\right| \phi_{l}\right)\right)\left[\bar{a} \bar{b}\left(\overline{\mathbf{A}}_{k l}^{-1} \overline{\mathbf{J}}_{11} \overline{\mathbf{A}}_{k l}^{-1} \overline{\mathbf{J}}_{g} \overline{\mathbf{A}}_{k l}^{-1}\right)_{\alpha \beta}+\bar{a} \bar{b}\left(\overline{\mathbf{A}}_{k l}^{-1} \overline{\mathbf{J}}_{g} \overline{\mathbf{A}}_{k l}^{-1} \overline{\mathbf{J}}_{11} \overline{\mathbf{A}}_{k l}^{-1}\right)_{\alpha \beta}-\bar{a} \bar{c}\left(\overline{\mathbf{A}}_{k l}^{-1} \overline{\mathbf{J}}_{g} \overline{\mathbf{A}}_{k l}^{-1}\right)_{\alpha \beta}-\bar{b} \bar{c}\left(\overline{\mathbf{A}}_{k l}^{-1} \overline{\mathbf{J}}_{11} \overline{\mathbf{A}}_{k l}^{-1}\right)_{\alpha \beta}\right]$ $=-\frac{1}{2}\left\langle\phi_{k}\left|r_{1}^{-q}\right| \phi_{l}\right\rangle\left[\frac{m_{m k}-q}{\bar{a}} \operatorname{tr}[\overline{a l a} \overline{\mathbf{C}}]+\operatorname{tr}\left[\overline{\mathbf{A}}_{k l}^{-1} \overline{\mathbf{C}}\right]\right]$

$\left(\bar{a} \bar{b}\left(\operatorname{tr}\left[\overline{\mathbf{A}}_{k l}^{-1} \overline{\mathbf{J}}_{g} \overline{\mathbf{A}}_{k l}^{-1} \overline{\mathbf{J}}_{11} \overline{\mathbf{A}}_{k l}^{-1} \overline{\mathbf{B}}\right]+\operatorname{tr}\left[\overline{\mathbf{A}}_{k l}^{-1} \overline{\mathbf{J}}_{11} \overline{\mathbf{A}}_{k l}^{-1} \overline{\mathbf{J}}_{g} \overline{\mathbf{A}}_{k l}^{-1} \overline{\mathbf{B}}\right]\right)-\bar{a} \bar{c} \operatorname{tr}\left[\mathbf{A}_{k l}^{-1} \overline{\mathbf{J}}_{g} \overline{\mathbf{A}}_{k l}^{-1} \overline{\mathbf{B}}\right]-\bar{b} \bar{c} \operatorname{tr}\left[\overline{\mathbf{A}}_{k l}^{-1} \overline{\mathbf{J}}_{11} \overline{\mathbf{A}}_{k l}^{-1} \overline{\mathbf{B}}\right]\right)$,

- $\overline{\mathbf{B}}^{\alpha \beta} \overline{\mathbf{C}}^{\rho \gamma}\left(\partial_{\rho \gamma} \frac{1}{\bar{a}^{2} \bar{b}^{2} \sqrt{\bar{b}}}\right)\left[\bar{a} \bar{b}\left(\overline{\mathbf{A}}_{k l}^{-1} \overline{\mathbf{J}}_{11} \overline{\mathbf{A}}_{k l}^{-1} \overline{\mathbf{J}}_{g} \overline{\mathbf{A}}_{k l}^{-1}\right)_{\alpha \beta}+\bar{a} \bar{b}\left(\overline{\mathbf{A}}_{k l}^{-1} \overline{\mathbf{J}}_{g} \overline{\mathbf{A}}_{k l}^{-1} \overline{\mathbf{J}}_{11} \overline{\mathbf{A}}_{k l}^{-1}\right)_{\alpha \beta}-\bar{a} \bar{c}\left(\overline{\mathbf{A}}_{k l}^{-1} \overline{\mathbf{J}}_{g} \overline{\mathbf{A}}_{k l}^{-1}\right)_{\alpha \beta}-\bar{b} \bar{c}\left(\overline{\mathbf{A}}_{k l}^{-1} \overline{\mathbf{J}}_{11} \overline{\mathbf{A}}_{k l}^{-1} \overline{\mathbf{A}}_{k l}^{-1}\right)_{\alpha \beta}\right]$ $=\frac{1}{\sqrt{\bar{b}}} \frac{1}{\bar{a}^{2} \bar{b}^{2}}\left[\frac{5}{2} \frac{1}{\bar{b}} \operatorname{tr}\left[\overline{\mathbf{A}}_{k l}^{-1} \overline{\mathbf{J}}_{g} \overline{\mathbf{A}}_{k l}^{-1} \overline{\mathbf{C}}\right]+2 \frac{1}{\bar{a}} \operatorname{tr}\left[\overline{\mathbf{A}}_{k l}^{-1} \overline{\mathbf{J}}_{11} \overline{\mathbf{A}}_{k l}^{-1} \overline{\mathbf{C}}\right]\right]$

$\times\left[\bar{a} \bar{b}\left(\operatorname{tr}\left[\overline{\mathbf{A}}_{k l}^{-1} \overline{\mathbf{J}}_{g} \overline{\mathbf{A}}_{k l}^{-1} \overline{\mathbf{J}}_{11} \overline{\mathbf{A}}_{k l}^{-1} \overline{\mathbf{B}}\right]+\operatorname{tr}\left[\overline{\mathbf{A}}_{k l}^{-1} \overline{\mathbf{J}}_{11} \overline{\mathbf{A}}_{k l}^{-1} \overline{\mathbf{J}}_{g} \overline{\mathbf{A}}_{k l}^{-1} \overline{\mathbf{B}}\right]\right)-\bar{a} \bar{c} \operatorname{tr}\left[\overline{\mathbf{A}}_{k l}^{-1} \overline{\mathbf{J}}_{g} \overline{\mathbf{A}}_{k l}^{-1} \overline{\mathbf{B}}\right]-\bar{b} \bar{c} \operatorname{tr}\left[\overline{\mathbf{A}}_{k l}^{-1} \overline{\mathbf{J}}_{11} \overline{\mathbf{A}}_{k l}^{-1} \overline{\mathbf{B}}\right]\right]$,

- $\quad \overline{\mathbf{B}}^{\alpha \beta} \overline{\mathbf{C}}^{\rho \gamma}\left(\partial_{\rho \gamma} \frac{\bar{c}}{\bar{a} \bar{b}}\right)\left[\bar{a} \bar{b}\left(\overline{\mathbf{A}}_{k l}^{-1} \overline{\mathbf{J}}_{11} \overline{\mathbf{A}}_{k l}^{-1} \overline{\mathbf{J}}_{g} \overline{\mathbf{A}}_{k l}^{-1}\right)_{\alpha \beta}+\bar{a} \bar{b}\left(\overline{\mathbf{A}}_{k l}^{-1} \overline{\mathbf{J}}_{g} \overline{\mathbf{A}}_{k l}^{-1} \overline{\mathbf{J}}_{11} \overline{\mathbf{A}}_{k l}^{-1}\right)_{\alpha \beta}-\bar{a} \bar{c}\left(\overline{\mathbf{A}}_{k l}^{-1} \overline{\mathbf{J}}_{g} \overline{\mathbf{A}}_{k l}^{-1}\right)_{\alpha \beta}-\bar{b} \bar{c}\left(\overline{\mathbf{A}}_{k l}^{-1} \overline{\mathbf{J}}_{11} \overline{\mathbf{A}}_{k l}^{-1}\right)_{\alpha \beta}\right]$ $=-\frac{1}{\bar{a}^{2} \bar{b}^{2}}\left(\bar{a} \bar{b}\left(\operatorname{tr}\left[\overline{\mathbf{A}}_{k l}^{-1} \overline{\mathbf{J}}_{g} \overline{\mathbf{A}}_{k l}^{-1} \overline{\mathbf{J}}_{11} \overline{\mathbf{A}}_{k l}^{-1} \overline{\mathbf{C}}\right]+\operatorname{tr}\left[\overline{\mathbf{A}}_{k l}^{-1} \overline{\mathbf{J}}_{11} \overline{\mathbf{A}}_{k l}^{-1} \overline{\mathbf{J}}_{g} \overline{\mathbf{A}}_{k l}^{-1} \overline{\mathbf{C}}\right]\right)-\bar{c} \bar{b} \operatorname{tr}\left[\overline{\mathbf{A}}_{k l}^{-1} \overline{\mathbf{J}}_{11} \overline{\mathbf{A}}_{k l}^{-1} \overline{\mathbf{C}}\right]-\bar{c} \bar{a} \operatorname{tr}\left[\overline{\mathbf{A}}_{k l}^{-1} \overline{\mathbf{J}}_{g} \overline{\mathbf{A}}_{k l}^{-1} \overline{\mathbf{C}}\right]\right)$ $\times\left(\bar{a} \bar{b}\left(\operatorname{tr}\left[\overline{\mathbf{A}}_{k l}^{-1} \overline{\mathbf{J}}_{g} \overline{\mathbf{A}}_{k l}^{-1} \overline{\mathbf{J}}_{11} \overline{\mathbf{A}}_{k l}^{-1} \overline{\mathbf{B}}\right]+\operatorname{tr}\left[\overline{\mathbf{A}}_{k l}^{-1} \overline{\mathbf{J}}_{11} \overline{\mathbf{A}}_{k l}^{-1} \overline{\mathbf{J}}_{g} \overline{\mathbf{A}}_{k l}^{-1} \overline{\mathbf{B}}\right]\right)-\bar{a} \bar{c} \operatorname{tr}\left[\overline{\mathbf{A}}_{k l}^{-1} \overline{\mathbf{J}}_{g} \overline{\mathbf{A}}_{k l}^{-1} \overline{\mathbf{B}}\right]-\bar{b} \bar{c} \operatorname{tr}\left[\overline{\mathbf{A}}_{k l}^{-1} \overline{\mathbf{J}}_{11} \overline{\mathbf{A}}_{k l}^{-1} \overline{\mathbf{B}}\right]\right)$,

- $\overline{\mathbf{B}}^{\alpha \beta} \overline{\mathbf{C}}^{\rho \gamma} \partial_{\rho \gamma}\left\{\bar{a} \bar{b}\left(\left(\overline{\mathbf{A}}_{k l}^{-1} \overline{\mathbf{J}}_{11} \overline{\mathbf{A}}_{k l}^{-1} \overline{\mathbf{J}}_{g} \overline{\mathbf{A}}_{k l}^{-1}\right)_{\alpha \beta}+\left(\overline{\mathbf{A}}_{k l}^{-1} \overline{\mathbf{J}}_{g} \overline{\mathbf{A}}_{k l}^{-1} \overline{\mathbf{J}}_{11} \overline{\mathbf{A}}_{k l}^{-1}\right)_{\alpha \beta}\right)-\bar{a} \bar{c}\left(\overline{\mathbf{A}}_{k l}^{-1} \overline{\mathbf{J}}_{g} \overline{\mathbf{A}}_{k l}^{-1}\right)_{\alpha \beta}-\bar{b} \bar{c}\left(\overline{\mathbf{A}}_{k l}^{-1} \overline{\mathbf{J}}_{11} \overline{\mathbf{A}}_{k l}^{-1}\right)_{\alpha \beta}\right\}$

$$
\begin{aligned}
= & -\bar{a} \operatorname{tr}\left[\overline{\mathbf{A}}_{k l}^{-1} \overline{\mathbf{J}}_{g} \overline{\mathbf{A}}_{k l}^{-1} \overline{\mathbf{C}}\right]\left(\operatorname{tr}\left[\overline{\mathbf{A}}_{k l}^{-1} \overline{\mathbf{J}}_{11} \overline{\mathbf{A}}_{k l}^{-1} \overline{\mathbf{J}}_{g} \overline{\mathbf{A}}_{k l}^{-1} \overline{\mathbf{B}}\right]+\operatorname{tr}\left[\overline{\mathbf{A}}_{k l}^{-1} \overline{\mathbf{J}}_{g} \overline{\mathbf{A}}_{k l}^{-1} \overline{\mathbf{J}}_{11} \overline{\mathbf{A}}_{k l}^{-1} \overline{\mathbf{B}}\right]\right)+\bar{a} \operatorname{tr}\left[\overline{\mathbf{A}}_{k l}^{-1} \overline{\mathbf{J}}_{g} \overline{\mathbf{A}}_{k l}^{-1} \overline{\mathbf{B}}^{\prime}\right] \operatorname{tr}\left[\overline{\mathbf{A}}_{k l}^{-1} \overline{\mathbf{J}}_{g} \overline{\mathbf{A}}_{k l}^{-1} \overline{\mathbf{J}}_{11} \overline{\mathbf{A}}_{k l}^{-1} \overline{\mathbf{C}}\right] \\
& \left.+\operatorname{tr}\left[\overline{\mathbf{A}}_{k l}^{-1} \overline{\mathbf{J}}_{11} \overline{\mathbf{A}}_{k l}^{-1} \overline{\mathbf{J}}_{g} \overline{\mathbf{A}}_{k l}^{-1} \overline{\mathbf{C}}\right]\right)-\bar{b} \operatorname{tr}\left[\overline{\mathbf{A}}_{k l}^{-1} \overline{\mathbf{J}}_{11} \overline{\mathbf{A}}_{k l}^{-1} \overline{\mathbf{C}}\right]\left(\operatorname{tr}\left[\overline{\mathbf{A}}_{k l}^{-1} \overline{\mathbf{J}}_{11} \overline{\mathbf{A}}_{k l}^{-1} \overline{\mathbf{J}}_{g} \overline{\mathbf{A}}_{k l}^{-1} \overline{\mathbf{B}}\right]+\operatorname{tr}\left[\overline{\mathbf{A}}_{k l}^{-1} \overline{\mathbf{J}}_{g} \overline{\mathbf{A}}_{k l}^{-1} \overline{\mathbf{J}}_{11} \overline{\mathbf{A}}_{k l}^{-1} \overline{\mathbf{B}}\right]\right)+\bar{b} \operatorname{tr}\left[\overline{\mathbf{A}}_{k l}^{-1} \overline{\mathbf{J}}_{11} \overline{\mathbf{A}}_{k l}^{-1} \overline{\mathbf{B}}\right]
\end{aligned}
$$




$$
\begin{aligned}
& \times\left(\operatorname{tr}\left[\overline{\mathbf{A}}_{k l}^{-1} \overline{\mathbf{J}}_{g} \overline{\mathbf{A}}_{k l}^{-1} \overline{\mathbf{J}}_{11} \overline{\mathbf{A}}_{k l}^{-1} \overline{\mathbf{C}}\right]+\operatorname{tr}\left[\overline{\mathbf{A}}_{k l}^{-1} \overline{\mathbf{J}}_{11} \overline{\mathbf{A}}_{k l}^{-1} \overline{\mathbf{J}}_{g} \overline{\mathbf{A}}_{k l}^{-1} \overline{\mathbf{C}}\right]\right)+\bar{c}\left(\operatorname{tr}\left[\overline{\mathbf{A}}_{k l}^{-1} \overline{\mathbf{J}}_{g} \overline{\mathbf{A}}_{k l}^{-1} \overline{\mathbf{C}}\right] \operatorname{tr}\left[\overline{\mathbf{A}}_{k l}^{-1} \overline{\mathbf{J}}_{11} \overline{\mathbf{A}}_{k l}^{-1} \overline{\mathbf{B}}\right]\right. \\
& \left.+\operatorname{tr}\left[\overline{\mathbf{A}}_{k l}^{-1} \overline{\mathbf{J}}_{11} \overline{\mathbf{A}}_{k l}^{-1} \overline{\mathbf{C}}\right] \operatorname{tr}\left[\overline{\mathbf{A}}_{k l}^{-1} \overline{\mathbf{J}}_{g} \overline{\mathbf{A}}_{k l}^{-1} \overline{\mathbf{B}}\right]\right)-\bar{a} \bar{b}\left(\operatorname{tr}\left[\mathbf{A}_{k l}^{-1} \overline{\mathbf{B}}_{k l}^{-1} \overline{\mathbf{J}}_{g} \overline{\mathbf{A}}_{k l}^{-1} \overline{\mathbf{J}}_{11} \overline{\mathbf{A}}_{k l}^{-1} \overline{\mathbf{C}}^{\prime}\right]+\operatorname{tr}\left[\overline{\mathbf{A}}_{k l}^{-1} \overline{\mathbf{J}}_{11} \overline{\mathbf{A}}_{k l}^{-1} \overline{\mathbf{B}}_{k l}^{-1} \overline{\mathbf{J}}_{g} \overline{\mathbf{A}}_{k l}^{-1} \overline{\mathbf{C}}^{\prime}\right]\right. \\
& \left.+\operatorname{tr}\left[\mathbf{A}_{k l}^{-1} \overline{\mathbf{J}}_{g} \overline{\mathbf{A}}_{k l}^{-1} \overline{\mathbf{J}}_{11} \overline{\mathbf{A}}_{k l}^{-1} \overline{\mathbf{B}}_{k l} \overline{\mathbf{A}}_{k l}^{-1} \overline{\mathbf{C}}^{\prime}\right]\right)-\bar{a} \bar{b}\left(\operatorname{tr}\left[\overline{\mathbf{A}}_{k l}^{-1} \overline{\mathbf{B}} \overline{\mathbf{A}}_{k l}^{-1} \overline{\mathbf{J}}_{11} \overline{\mathbf{A}}_{k l}^{-1} \overline{\mathbf{J}}_{g} \overline{\mathbf{A}}_{k l}^{-1} \overline{\mathbf{C}}^{\prime}\right]+\operatorname{tr}\left[\overline{\mathbf{A}}_{k l}^{-1} \overline{\mathbf{J}}_{g} \overline{\mathbf{A}}_{k l}^{-1} \overline{\mathbf{B}}_{k l}^{-1} \overline{\mathbf{J}}_{11} \overline{\mathbf{A}}_{k l}^{-1} \overline{\mathbf{C}}^{\prime}\right]\right. \\
& \left.+\operatorname{tr}\left[\overline{\mathbf{A}}_{k l}^{-1} \overline{\mathbf{J}}_{11} \overline{\mathbf{A}}_{k l}^{-1} \overline{\mathbf{J}}_{g} \overline{\mathbf{A}}_{k l}^{-1} \overline{\mathbf{B}} \overline{\mathbf{A}}_{k l}^{-1} \overline{\mathbf{C}}^{\prime}\right]\right)+\bar{a} \bar{c} \operatorname{tr}\left[\overline{\mathbf{A}}_{k l}^{-1} \overline{\mathbf{B}} \overline{\mathbf{A}}_{k l}^{-1} \overline{\mathbf{J}}_{g} \overline{\mathbf{A}}_{k l}^{-1} \overline{\mathbf{C}}^{\prime}\right]+\operatorname{tr}\left[\overline{\mathbf{A}}_{k l}^{-1} \overline{\mathbf{J}}_{g} \overline{\mathbf{A}}_{k l}^{-1} \overline{\mathbf{B}}_{k l}^{-1} \overline{\mathbf{A}}_{k l}^{-1} \overline{\mathbf{C}}^{\prime}\right]+\bar{b} \bar{c} \operatorname{tr}\left[\overline{\mathbf{A}}_{k l}^{-1} \overline{\mathbf{B}}_{k l}^{-1} \overline{\mathbf{J}}_{11} \overline{\mathbf{A}}_{k l}^{-1} \overline{\mathbf{C}}^{\prime}\right] \\
& \left.+\operatorname{tr}\left[\overline{\mathbf{A}}_{k l}^{-1} \overline{\mathbf{J}}_{11} \overline{\mathbf{A}}_{k l}^{-1} \overline{\mathbf{B}} \overline{\mathbf{A}}_{k l}^{-1} \overline{\mathbf{C}}^{\prime}\right]\right) .
\end{aligned}
$$

This concludes the derivation of the matrix elements of the $\hat{H}_{o o}$ operator.

${ }^{1}$ W. E. Coswell and G. P. Lepage, Phys. Lett. B 167, 437 (1986).

${ }^{2}$ T. Kinoshita and M. Nio, Phys. Rev. D 53, 4909 (1996).

${ }^{3}$ H. A. Bethe and E. E. Salpeter, Quantum Mechanics of One- and TwoElectron (Plenum, New York, 1977).

${ }^{4}$ M. Stanke, D. Kedziera, S. Bubin, and L. Adamowicz, Phys. Rev. Lett. 99, 043001 (2007).

${ }^{5}$ M. Stanke, D. Kẹdziera, S. Bubin, and L. Adamowicz, Phys. Rev. A 75, 052510 (2007).

${ }^{6}$ M. Stanke, D. Kędziera, S. Bubin, and L. Adamowicz, J. Chem. Phys. 127, 134107 (2007).

${ }^{7}$ K. Pachucki and J. Komasa, J. Chem. Phys. 125, 204304 (2006).

${ }^{8}$ K. Pachucki and J. Komasa, Phys. Rev. A 73, 052502 (2006).

${ }^{9}$ K. Pachucki and J. Komasa, Phys. Rev. Lett. 92, 213001 (2004).

${ }^{10}$ M. Cafiero, S. Bubin, and L. Adamowicz, Phys. Chem. Chem. Phys. 5, 1491 (2003).

${ }^{11}$ S. Bubin, M. Cafiero, and L. Adamowicz, Adv. Chem. Phys. 131, 377 (2005).

${ }^{12}$ D. B. Kinghorn and L. Adamowicz, J. Chem. Phys. 110, 7166 (1999).

${ }^{13}$ D. B. Kinghorn and L. Adamowicz, Phys. Rev. Lett. 83, 2541 (1999).

${ }^{14}$ S. Bubin and L. Adamowicz, J. Chem. Phys. 118, 3079 (2003).

${ }^{15}$ M. Pavanello, S. Bubin, M. Molski, and L. Adamowicz, J. Chem. Phys 123, 104306 (2005).

${ }^{16}$ D. Kedziera, M. Stanke, S. Bubin, M. Barysz, and L. Adamowicz, J. Chem. Phys. 125, 084303 (2006).

${ }^{17}$ M. Stanke, D. Kedziera, S. Bubin, M. Barysz, and L. Adamowicz, Phys. Rev. Lett. 96, 233002 (2006).

${ }^{18}$ G. Tarczay, A. G. Császár, W. Klopper, and H. M. Quiney, Mol. Phys.
99, 1769 (2001).

${ }^{19}$ O. L. Polyansky, A. G. Császár, S. V. Shirin, N. F. Zobov, P. Barletta, J. Tennyson, D. W. Schwenke, and P. J. Knowles, Science 299, 539 (2003).

${ }^{20}$ D. Kȩdziera, M. Stanke, S. Bubin, M. Barysz, and L. Adamowicz, J. Chem. Phys. 125, 014318 (2006).

${ }^{21}$ S. Bubin, M. Stanke, D. Kędziera, and L. Adamowicz, Phys. Rev. A 76, 022512 (2007).

${ }^{22}$ I. Dabrowski, Can. J. Phys. 62, 1639 (1984).

${ }^{23}$ See CODATA 2002 recommended values and NIST Physical Reference Data at http://physics.nist.gov/

${ }^{24}$ J. L. Dunham, Phys. Rev. 41, 713, (1932); 41, 721 (1932).

${ }^{25}$ D. K. Veirs and G. M. Rosenblatt, J. Mol. Spectrosc. 121, 401 (1987).

${ }^{26}$ D. E. Jennings, A. Weber, and J. W. Brault, Appl. Opt. 25, 284 (1986).

${ }^{27}$ U. Fink, T. A. Wigging, and D. H. Rank, J. Mol. Spectrosc. 18, 384 (1965).

${ }^{28}$ B. P. Stoicheff, Can. J. Phys. 35, 730 (1957).

${ }^{29}$ E. C. Looi, J. C. Stryland, and H. L. Welsh, Can. J. Phys. 56, 1102 (1987).

${ }^{30}$ P. J. Brannon, C. H. Church, and C. W. Peters, J. Mol. Spectrosc. 27, 44 (1968).

${ }^{31}$ J. Geoffrey, G. J. Germann, and J. J. Valentini, J. Phys. Chem. 92, 3792 (1987).

${ }^{32}$ J. V. Foltz, D. H. Rank, and T. A. Wiggins, J. Mol. Spectrosc. 27, 44 (1968).

${ }^{33}$ H. L. Buijs and H. P. Gush, Can. J. Phys. 49, 2366 (1971).

${ }^{34}$ L. Wolniewicz, J. Chem. Phys. 99, 1851 (1993).

${ }^{35} \mathrm{~L}$. Wolniewicz (private communication).

${ }^{36}$ D. B. Kinghorn, Int. J. Quantum Chem. 57, 141 (1996).

${ }^{37}$ S. Bubin, M. Stanke, D. Kedziera, and L. Adamowicz, Phys. Rev. A 75, 062504 (2007).

${ }^{38}$ E. Bednarz, S. Bubin, and L. Adamowicz, Mol. Phys. 103, 1169 (2005). 